\title{
THE RESOLVENT PARAMETRIX OF THE GENERAL ELLIPTIC LINEAR DIFFERENTIAL OPERATOR: A CLOSED FORM FOR THE INTRINSIC SYMBOL
}

\author{
S. A. FULLING AND G. KENNEDY
}

\begin{abstract}
Nonrecursive, explicit expressions are obtained for the term of arbitrary order in the asymptotic expansion of the intrinsic symbol of a resolvent parametrix of an elliptic linear differential operator, of arbitrary order and algebraic structure, which acts on sections of a vector bundle over a manifold. Results for the conventional symbol are included as a special case.
\end{abstract}

1. Introduction. As is well known, the resolvent operator, $(A-\lambda)^{-1}$, plays a central role in the functional analysis associated with an elliptic linear differential operator $A$. In particular, from it one can easily obtain the corresponding heat operator, $e^{-t A}$, for $t \in \mathbf{R}_{+}$and semibounded $A$. Furthermore, detailed knowledge of the terms in the asymptotic expansions of the integral kernels of the resolvent and heat operators is of great value in calculating the asymptotics of eigenvalues and spectral functions $[\mathbf{1 2}, \mathbf{2 5}, \mathbf{2}, \mathbf{3}, \mathbf{3 3}]$; partial solutions of inverse problems [26, 15]; indices of Fredholm operators $[\mathbf{1}, \mathbf{1 9}, \mathbf{2 0}]$; and various physical quantities, including specific heats [4], partition functions [49], renormalized effective actions $[38,14,34,45,8]$, and renormalized energy-momentum tensors $[9,43,44]$. (The references given here are merely representative.) It is important, therefore, to have available an efficient and general method for calculating such terms. The present paper addresses this topic for the resolvent operator, or, more precisely, a resolvent parametrix; later papers will treat the heat operator.

Before the advent of pseudodifferential-operator techniques, parametrices of differential operators were constructed directly in "coordinate space", i.e., without the use of the Fourier transform $[\mathbf{2 3}, \mathbf{3 2}, \mathbf{3 1}]$, and this approach is still commonly used in the physics (and other) literature $[\mathbf{1 4}, \mathbf{3 6}, \mathbf{9}, \mathbf{1 0}]$. In geometrical contexts it can be implemented in a "manifestly covariant" manner, so that its results are automatically expressed in terms of intrinsically defined objects, such as tensors, covariant derivatives, and curvature; however, its applicability appears to be limited to second-order operators with scalar principal symbol. (A differential operator which acts on sections of a vector bundle is said to have scalar principal symbol if its leading-order coefficient is a tensor multiple of the identity endomorphism of the bundle.) Thus, it is unsuitable for handling the fourth-order operators (with scalar principal symbol) and second-order operators with nonscalar principal

Received by the editors June 8, 1987.

1980 Mathematics Subject Classification (1985 Revision). Primary 35S99; Secondary 35J30, $35 \mathrm{~J} 45,58 \mathrm{G} 15$. 
symbol which arise both in elasticity theory [4] and in certain quantum gravity theories $[\mathbf{1 1}, \mathbf{5}]$. (It was the unsuitability for the latter application which motivated the present work.)

Nowadays, the symbolic calculus of pseudodifferential operators $[\mathbf{3 5}, \mathbf{4 2}, \mathbf{4 1}, \mathbf{2 9}]$ is a much more widely used technique among mathematicians $[\mathbf{3 9}, \mathbf{2 2}, \mathbf{1 8}, \mathbf{3 7}]$, although it has so far remained largely unknown in the physics and applied mathematics communities, even though it has much to offer them. Its importance is due, in part, to the generality of the operators to which it may be applied; even operators with nonscalar principal symbol can be handled, although not without encountering grave computational complexities $[\mathbf{1 8}, \mathbf{4 7}, \mathbf{4 8}]$. However, the conventional symbolic calculus on manifolds has a defect from which the older technique is free: Because it is based on Fourier analysis in local coordinate charts, the results which it produces are expressed in terms of nontensorial functions and their partial derivatives; it is tedious, inefficient, and inelegant to rewrite these in an intrinsic way in terms of tensors and covariant derivatives, especially in high-order calculations, where the number of algebraically independent quantities is very large. This has not prevented mathematicians from proving many valuable theorems on the basis of only a general understanding of the nature of the terms in parametrical series. In fact, they have often been deterred from performing more explicit calculations of these terms by their lack of a need for such detail and by the computational complexity involved. (When explicit calculations have been performed, they have not gone very far beyond the level already reached by coordinate-space methods.) Physicists and applied mathematicians, on the other hand, could make good use of such explicit results - especially, results which are expressed intrinsically and which are calculated to high order. (The current investigations by physicists of KaluzaKlein-type theories on manifolds of dimension 10 or more $[\mathbf{1 3}, \mathbf{3 0}, \mathbf{5 0}]$ would benefit from certain calculations of order proportional to the dimension.)

There is, in fact, an alternative symbolic calculus which combines the advantages of both these approaches generality and an intrinsic formalism. This is the intrinsic symbolic calculus, which was pioneered by Widom $[\mathbf{4 6}, \mathbf{4 7}]$ and has received contributions from Drager [16], Allard (unpublished), and Kennedy [27] (see also the work of Bokobza-Haggiag [6], Getzler [17], Smith [40], and Bunch and Parker [7]). Unfortunately, it is not yet widely known, even among mathematicians, and has so far remained calculationally underdeveloped. We hope that the present paper and its sequels will help to turn the intrinsic calculus into an efficient calculational tool.

The central idea of the intrinsic calculus is to replace the coordinate-dependent object $\langle\xi, y-x\rangle$, which appears in the formulas of the conventional calculus, by the intrinsically defined phase function $\phi(\xi, y)=\left\langle\xi, \exp _{x}^{-1}(y)\right\rangle$, where $\xi \in T_{x}^{*}(M)$, $y \in M$, and $\exp _{x}$ is the exponential mapping at $x \in M$, which is derived from a given connection on the cotangent bundle $T^{*}(M)$ of the manifold $M$. With $\phi$ and, in the case of operators acting on sections of a vector bundle $E$, a second quantity, $\tau^{E}$, which is essentially parallel transport with respect to a given connection on $E$, one can introduce an intrinsic definition of the symbol of a pseudodifferential operator on $M$, which has the property that the complete symbol is a homomorphism-valued function on $T^{*}(M)$. (In the conventional definition, only the principal part of the 
symbol has this interpretation.) The resulting symbolic calculus involves covariant rather than partial derivatives and hence provides the sought-after intrinsic formalism for parametrical calculations.

In $\S 2$ we present a careful construction of $\phi$ and $\tau^{E}$ and state their important properties. The definition of the intrinsic symbol and the essentials of the symbolic calculus, including the formula for the symbol of a product, are to be found in $\S 3$, which also contains certain notational remarks of relevance to later sections.

In $\S 4$ we embark on the quest for an explicit, closed-form expression for the term $b_{s}$ of arbitrary order $s$ in the asymptotic expansion of the intrinsic symbol of a resolvent parametrix. The solution of the recursion relation which these terms satisfy, Proposition 4.2, sets us on our way, and the journey to the main results, Theorems 4.1 and 4.2, mostly involves navigating through some heavy algebra and taming some monstrous summations over multi-indices. In much of that section we permit $A$ to be a classical pseudodifferential operator; Theorem 4.1 expresses $b_{s}$ in terms of covariant derivatives of the terms $\left\{a_{r}\right\}$ in the asymptotic expansion of the intrinsic symbol of $A$, and covariant derivatives of $\phi$ and $\tau^{E}$. (It is through the latter quantities that the influence of the manifold curvature and torsion and the bundle curvature is made manifest, although we shall not make this explicit in our results.) Eventually, in Theorem 4.2, we specialize to the case of a differential operator and rewrite this result in terms of covariant derivatives of its coefficient tensors.

In the formulas of $\S 4$ we have striven to write the various summations over multiindices in as compact and illuminating a way as possible. However, it will be useful for later applications to introduce a canonical ordering of these summations. Such an ordering is performed in $\S 5$.

Upon specialization to flat connections, the intrinsic calculus reduces to the conventional calculus. In $\S 6$ we consider this reduction in the case of Theorem 4.2 and thereby obtain Theorem 6.1, which expresses the general term in the asymptotic expansion of the conventional symbol of a resolvent parametrix (relative to a choice of local coordinate chart and bundle frame) in terms of partial derivatives of the coefficient functions of the differential operator.

$\S 7$ contains some concluding remarks.

This work was supported, in part, by NSF grants PHY 79-15229 and PHY 8403575 and by NATO Fellowship B/RF 5638. Preliminary studies began in the spring of 1981 when the authors enjoyed the hospitality of the Institute for Theoretical Physics at the University of California, Santa Barbara. We thank S. M. Christensen for instigating and encouraging the investigations of this paper and its sequels, L. D. Drager for calling the intrinsic symbolic calculus to our attention and providing a copy of his dissertation, and L. D. Drager, H. Widom, and W. K. Allard for kindly and patiently explaining their results.

2. Linearization and local transport. Let $M$ be a $C^{\infty}$ paracompact real manifold of dimension $d<\infty$ and let $E$ be a $C^{\infty}$ (real or complex) vector bundle with base $M$ and projection $\pi: E \rightarrow M$. In this section we shall introduce the concepts of linearization of $M$ and local transport of $E$, both of which are fundamental to the definition of the intrinsic symbol of a pseudodifferential operator which acts on smooth sections of $E$. Although this may be done in a quite general way, as in the work of Bokobza-Haggiag [6], a more constructive approach, due to 
Widom [46, 47] and Drager [16], is possible if both $E$ and $T^{*}(M)$ are equipped with connections. The latter route will be followed here, although our exposition will be brief; further details may be found in the above references or in [27].

Thus, let $E$ and $T^{*}(M)$ be equipped with $C^{\infty}$ connections, $\nabla: \Gamma^{\infty}(E) \rightarrow$ $\Gamma^{\infty}\left(E \otimes T^{*}(M)\right)$ and $\nabla: \Gamma^{\infty}\left(T^{*}(M)\right) \rightarrow \Gamma^{\infty}\left(T^{*}(M) \otimes T^{*}(M)\right)$, and let any tensorproduct bundle $H$ formed from $E$ or $T^{*}(M)$ or their duals inherit the corresponding tensor-product connection, $\nabla: \Gamma^{\infty}(H) \rightarrow \Gamma^{\infty}\left(H \otimes T^{*}(M)\right)$. For all $m \in \mathbf{Z}_{+}$, $\nabla^{m}: \Gamma^{\infty}(H) \rightarrow \Gamma^{\infty}\left(H \otimes\left(\bigotimes^{m}\left(T^{*}(M)\right)\right)\right)$ will denote the $m$-compose $\nabla \circ \nabla \circ \cdots \circ \nabla$, and $\hat{\nabla}^{m}: \Gamma^{\infty}(H) \rightarrow \Gamma^{\infty}\left(H \otimes S\left(\bigotimes^{m}\left(T^{*}(M)\right)\right)\right)$ the result of $\nabla^{m}$ followed by the symmetrization $S: \bigotimes^{m}\left(T^{*}(M)\right) \rightarrow S\left(\bigotimes^{m}\left(T^{*}(M)\right)\right)$.

The connections define the exponential and parallel-transport mappings in the usual way (see, for example, the treatises of Kobayashi and Nomizu [28] or Helgason [24]). In particular, for each $x \in M$ there exist a normal neighborhood $N_{x}$ of 0 in $T_{x}(M)$ and a normal neighborhood $U_{x}$ of $x$ in $M$ such that the exponential mapping at $x, \exp _{x}: N_{x} \rightarrow U_{x}$, is a diffeomorphism. Moreover, for each $y \in U_{x}$ the parallel-transport mapping, $\tau_{x, y}^{E}: E_{x} \rightarrow E_{y}$, of fibers of $E$ (along the unique geodesic segment in $U_{x}$ which joins $x$ and $y$ ) is a well-defined isomorphism. Globally, we have the following well-known

PROPOSITION 2.1. There exists an open neighborhood $\Omega_{0}$ of the diagonal $\Delta$ in $M \times M$ such that the mapping defined by $\Omega_{0} \ni(x, y) \mapsto \exp _{x}^{-1}(y) \in T_{x}(M)$ is a diffeomorphism of $\Omega_{0}$ onto an open neighborhood of the zero section in $T(M)$.

We are now prepared to define the fundamental objects of this section.

DEFINITION 2.1. Let $\Omega_{0}$ be as in Proposition 2.1 and let $\alpha_{0} \in C^{\infty}(M \times M)$ have support contained in $\Omega_{0}$ and be equal to 1 on a neighborhood of $\Delta$. Natural extension (by 0 on $M \times M \backslash \Omega_{0}$ ) of $\Omega_{0} \ni(x, y) \mapsto \alpha_{0}(x, y) \exp _{x}^{-1}(y) \in T_{x}(M)$ defines a $C^{\infty}$ mapping $\Phi: M \times M \rightarrow T(M)$ such that the diagram

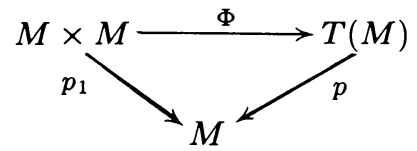

commutes, where $p_{i}$ is the projection on the $i$ th factor and $p: T(M) \rightarrow M$ is the canonical projection of $T(M)$. $\Phi$ will be called a connection-induced linearization of $M$.

DEFINITION 2.2. Let $\Phi$ be a connection-induced linearization of $M$ and let $\phi \in$ $C^{\infty}\left(T^{*}(M) \times M\right)$ be defined by $\phi(\xi, y)=\langle\xi, \Phi(p(\xi), y)\rangle$ for all $(\xi, y) \in T^{*}(M) \times M$, where $p: T^{*}(M) \rightarrow M$ is the canonical projection of $T^{*}(M)$. $\phi$ will be called the phase function of $M$ associated to $\Phi$.

DEFINITION 2.3. Let $\Omega_{0}$ be as in Proposition 2.1 and $\alpha_{0}$ as in Definition 2.1. Natural extension (by 0 on $M \times M \backslash \Omega_{0}$ ) of $\Omega_{0} \ni(x, y) \mapsto \alpha_{0}(x, y) \tau_{x, y}^{E} \in \operatorname{Hom}\left(E_{x}, E_{y}\right)$ defines the mapping $M \times M \ni(x, y) \mapsto \hat{\tau}_{x, y}^{E} \in \operatorname{Hom}\left(E_{x}, E_{y}\right)$. The $C^{\infty}$ mapping $\tau^{E}: E \times M \rightarrow E$ defined by $\tau^{E}(e, y)=\hat{\tau}_{\pi(e), y}^{E}(e)$ for all $(e, y) \in E \times M$ is such that the diagram

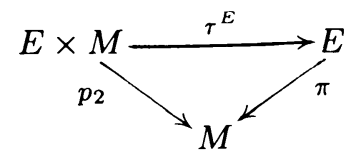

commutes. $\tau^{E}$ will be called a connection-induced local transport of $E$. 
REMARK 2.1. $\Phi$ is a linearization of $M$ and (modulo interchange of its arguments) $\tau^{E}$ is a local transport of $E$ according to the more general definitions of such objects given by Bokobza-Haggiag [6], who does not employ connections. Her terminology is adopted here, the qualifier "connection-induced" being appended when, as above, $\Phi$ and $\tau^{E}$ are constructed from the exponential and parallel-transport mappings derived from given connections.

The most important properties of $\Phi, \phi$, and $\tau^{E}$ are contained in the following propositions. Proofs of the first may be found in $[47,16$, or 27]; proofs of the second may be found in [6 or 27].

Proposition 2.2. Let $\Phi$ be a connection-induced linearization of $M, \phi$ the associated phase function, and $\tau^{E}$ a connection-induced local transport of $E$. Then

$$
\left(\hat{\nabla}^{m} \phi(\xi, \cdot)\right)(p(\xi))= \begin{cases}\xi & \text { if } m=1 \\ 0 & \text { if } m \neq 1\end{cases}
$$

and

$$
\left(\hat{\nabla}^{m} \tau^{E}(e, \cdot)\right)(\pi(e))= \begin{cases}e & \text { if } m=0 \\ 0 & \text { if } m \neq 0\end{cases}
$$

for all $m \in \mathbf{Z}_{+}, \xi \in T^{*}(M)$, and $e \in E$.

PROPOSITION 2.3. Let $\Phi$ be a connection-induced linearization of $M$ and $\tau^{E} a$ connection-induced local transport of $E$. There exists an open neighborhood $\Omega \subset \Omega_{0}$ of $\Delta$ which satisfies the following conditions:

(i) $\Phi \mid \Omega$ is a diffeomorphism of $\Omega$ onto an open neighborhood of the zero section in $T(M)$.

(ii) $\hat{\tau}_{x, y}^{E}$ is an isomorphism for each $(x, y) \in \Omega$.

(iii) $p_{1}: \bar{\Omega} \rightarrow M$ and $p_{2}: \bar{\Omega} \rightarrow M$ are proper mappings.

(iv) $\Omega$ is symmetric.

(v) $\Omega_{x}=\{y \in M:(x, y) \in \Omega\}$ is a (convex) normal neighborhood of each of its points for each $x \in M$.

DEFINITION 2.4. Let $\Omega$ be as in Proposition 2.3. $\Omega$ will be called a domain of $\Phi$ and $\tau^{E}$.

REMARK 2.2. We may, of course, choose $\Omega$ to be so close to $\Delta$ that $\alpha_{0}$ is equal to 1 on $\Omega$, in which case $\Phi(x, y)=\exp _{x}^{-1}(y)$ and $\hat{\tau}_{x, y}^{E}=\tau_{x, y}^{E}$ for all $(x, y) \in \Omega$. This will be assumed in the remainder of this paper.

We conclude this section with some miscellaneous remarks.

REMARK 2.3. In the work of Widom $[\mathbf{4 6}, \mathbf{4 7}]$, the objects corresponding to $\phi$ and $\tau^{E}$ are denoted by $l$ and $c$, respectively, and are defined in a slightly more general way than above as unspecified mappings having the jets (2.1) and (2.2). The present explicit construction in terms of the exponential and parallel-transport mappings is due to Drager [16], who, incidentally, denotes $\Phi$ by $v$. Both approaches yield the same intrinsic symbolic calculus for pseudodifferential operators, modulo smoothing.

REMARK 2.4. If $M$ is equipped with a metric $g$, then one may define $\sigma \in$ $C^{\infty}\left(\Omega_{0}\right)$ by requiring that $\sigma\left(x, x^{\prime}\right)$ equal half the square of the length of the geodesic segment which joins $x$ and $x^{\prime}$. This function plays an important role in the work of DeWitt $[\mathbf{1 4}]$ and Christensen $[\mathbf{9}, \mathbf{1 0}]$ on quantum field theory on manifolds. These 
authors employ a connection on $T^{*}(M)$ which is compatible with $g$ and denote by $\sigma_{\mu}\left(x, x^{\prime}\right)=g_{\mu \nu}(x) \sigma^{\nu}\left(x, x^{\prime}\right)$ the components, $\partial \sigma\left(x, x^{\prime}\right) / \partial x^{\mu}$, of $\operatorname{grad}_{x} \sigma\left(x, x^{\prime}\right)$ with respect to a local coordinate frame. (They also denote $\tau_{x, x^{\prime}}^{E}$ by $I\left(x^{\prime}, x\right)$.) The relationship with the objects of this section appears through the equation

$$
\exp _{x}^{-1}\left(x^{\prime}\right)=-\sigma^{\mu}\left(x, x^{\prime}\right) \frac{\partial}{\partial x^{\mu}}
$$

Thus, in terms of their notation, we may write

$$
\begin{gathered}
\Phi^{\mu}\left(x, x^{\prime}\right)=-\alpha_{0}\left(x, x^{\prime}\right) \sigma^{\mu}\left(x, x^{\prime}\right), \\
\phi\left(\xi, x^{\prime}\right)=-\alpha_{0}\left(x, x^{\prime}\right) \xi_{\mu} \sigma^{\mu}\left(x, x^{\prime}\right), \\
((\nabla \phi(\xi, \cdot))(x))_{\nu}=-\xi_{\mu}\left[\sigma_{\nu^{\prime}}^{\mu}\right],
\end{gathered}
$$

etc., where $\xi \in T_{x}^{*}(M)$. This link with $\sigma$ can be quite useful, as, for example, in the following remark. However, in the present work (as in $[\mathbf{4 6}, \mathbf{4 7}, \mathbf{1 6}, \mathbf{6}$, and 27]) no metric is assumed, and therefore $\sigma$ is undefined.

REMARK 2.5. As will be seen in the following sections, the formulas of the intrinsic symbolic calculus and the main results of this paper typically involve multiple covariant derivatives of $\phi$ and $\tau^{E}$ which, unlike those in (2.1) and (2.2), are not totally symmetrized. In fact, it is through such quantities that the influence of the manifold curvature and torsion and the bundle curvature is made manifest, and it is important, therefore, to have efficient calculational techniques which express them in terms of such geometrical objects. One method, which was devised by Widom $[46,47]$ and has recently been extended by Kennedy (unpublished), combines the Ricci identities with (2.1) and (2.2). Another, which is due to De Witt [14] and Christensen $[\mathbf{9}, \mathbf{1 0}]$, and which is possible only when $M$ is equipped with a metric, exploits the identity $\sigma^{\mu} \sigma_{\mu}=2 \sigma$ which is satisfied by the function $\sigma$ of the preceding remark. Although this method has a more restricted domain of applicability than the first, it is also more efficient. It has recently been extended by Schimming [53] and Rodionov and Taranov [52]; see also Fulling [51]. Yet another method has been given by Drager [16].

3. The intrinsic symbolic calculus. Let $F$ be a $C^{\infty}$ (real or complex) vector bundle with base $M$ and let $L^{l}(M, E, F)$ be the space of (standard) pseudodifferential operators $A: \Gamma_{0}^{\infty}(E) \rightarrow \Gamma^{\infty}(F)$ of order $l \in \mathbf{R}$. In this section we shall define the intrinsic symbol of $A$ and present the important results of the intrinsic symbolic calculus, in a formulation which differs slightly from that of Widom $[46,47]$ and Drager [16].

DEFINITION 3.1. Let $\Phi$ be a connection-induced linearization of $M, \phi$ the associated phase function, $\tau^{E}$ a connection-induced local transport of $E, \Omega$ a domain of $\Phi$ and $\tau^{E}$, and let $\alpha \in C^{\infty}(M \times M)$ have support contained in $\Omega$ and be equal to 1 on a neighborhood of $\Delta$. If $A \in L^{l}(M, E, F)$ then one can define a $C^{\infty}$ mapping $a: T^{*}(M) \rightarrow \operatorname{Hom}(E, F)$ such that the diagram

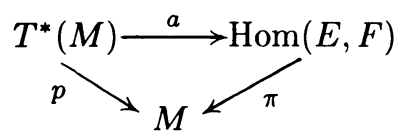

commutes, where $\pi: \operatorname{Hom}(E, F) \rightarrow M$ denotes the projection of the homomorphism bundle, by

$$
(a(\xi))(e)=A\left\{\alpha(p(\xi), \cdot) \tau^{E}(e, \cdot) e^{i \phi(\xi, \cdot)}\right\}(p(\xi))
$$


for all $\xi \in T^{*}(M)$ and $e \in E_{p(\xi)}$. a will be called the intrinsic symbol of $A$.

REMARK 3.1. This definition is originally due to Bokobza-Haggiag and in [6] is stated in terms of her more general definitions of linearization and local transport. Unfortunately, because her work is so general and, in particular, does not involve connections, the symbolic calculus which results is not very useful. Widom [47] employs the same definition and, by introducing connections, is able to develop a practical symbolic calculus.

REMARK 3.2. It follows from condition (iii) of Proposition 2.3 that $\alpha$ is properly supported. Hence the argument of $A$ in (3.2) belongs, as it should, to $\Gamma_{0}^{\infty}(E)$.

We shall denote by $S^{l}\left(T^{*}(M), \operatorname{Hom}(E, F)\right)$ the space of mappings a satisfying (3.1) whose component functions, relative to a local frame for $\operatorname{Hom}(E, F)$ and coordinate chart for $T^{*}(M)$, obey the usual inequality condition for symbols of order $l$. If $A \in L^{l}(M, E, F)$ and $a$ is defined according to (3.2) then $a \in S^{l}\left(T^{*}(M), \operatorname{Hom}(E, F)\right)$.

REMARK 3.3. Different choices of $\alpha, \phi$, and $\tau^{E}$ in (3.2) can alter $a$ by at most an element of $S^{-\infty}$. By the intrinsic symbol of $A$ we mean a representative of this equivalence class.

A useful feature of Definition 3.1 is that if $A$ is a differential operator then its intrinsic symbol is obtained by replacing $\nabla$ by $i \xi$. More precisely, we have the following proposition, which is a simple consequence of Proposition 2.2.

PROPOSITION 3.1. The intrinsic symbol of a differential operator

$$
A=\sum_{r=0}^{l} A_{r} \hat{\nabla}^{l-r}
$$

of order $l \geq 0$, where $A_{r} \in \Gamma^{\infty}\left(\operatorname{Hom}(E, F) \otimes S\left(\otimes^{l-r}(T(M))\right)\right)$, is $a=\sum_{r=0}^{l} a_{r}$, where $a_{r}(\xi)=\left(A_{r}(p(\xi))\right)\left(\bigotimes^{l-r}(i \xi)\right)$ for all $\xi \in T^{*}(M)$ and contraction with $\bigotimes^{l-r}(i \xi)$ is understood.

The formula for recovering an operator from its intrinsic symbol may be written in several ways. The version in the following proposition is due to Bokobza-Haggiag and is proved in [6]; others may be found in [27].

Proposition 3.2. Let $A \in L^{l}(M, E, F)$ have intrinsic symbol

$$
a \in S^{l}\left(T^{*}(M), \operatorname{Hom}(E, F)\right),
$$

as in Definition 3.1. Then

$$
\begin{aligned}
& A\left\{\alpha^{2}(x, \cdot) u\right\}(x) \\
& \quad=(2 \pi)^{-d} \int_{T_{x}^{*}(M)} a(\xi) \int_{\Omega_{x}} \alpha(x, y) \tau^{E}(u(y), x) e^{-i \phi(\xi, y)} \operatorname{det} \frac{\partial \Phi(x, y)}{\partial y} d y d \xi
\end{aligned}
$$

for all $u \in \Gamma_{0}^{\infty}(E)$ and $x \in M$. The expression on the right defines a properly supported pseudodifferential operator $\hat{A} \in L^{l}(M, E, F)$ such that $(\hat{A} u)(x)=$ $A\left\{\alpha^{2}(x, \cdot) u\right\}(x)$, and $\hat{A}$ differs from $A$ by a smoothing operator whose integral kernel vanishes on a neighborhood of $\Delta$.

REMARK 3.4. The integration over $y$ in (3.3) plays the role of the Fourier transform of $u$. 
An important ingredient for the development of the symbolic calculus is a definition of covariant differentiation of an intrinsic symbol. In that which follows, and in the remainder of this section, we let $F$ be equipped with a $C^{\infty}$ connection; $\operatorname{Hom}(E, F) \simeq E^{*} \otimes F$ then inherits the corresponding tensor-product connection.

DEFINITION 3.2. Let $a: T^{*}(M) \rightarrow \operatorname{Hom}(E, F)$ be a $C^{\infty}$ mapping satisfying (3.1). Differentiation along fibers of $T^{*}(M)$ will be denoted by $D$ and is given the usual vector-space definition:

$$
\left(\left(D^{m} a\right)(\xi)\right)(\eta, \ldots, \eta)=\left.\frac{d^{m}}{d t^{m}} a(\xi+t \eta)\right|_{t=0}
$$

for all $m \in \mathbf{Z}_{+}$and $\xi, \eta \in T^{*}(M)$ such that $p(\xi)=p(\eta)$. Differentiation along directions in the base will be denoted by $\nabla$ and is defined by

$$
\left(\hat{\nabla}^{m} a\right)(\xi)=\left(\hat{\nabla}^{m} a\left(\tau^{T^{*}(M)}(\xi, \cdot)\right)\right)(p(\xi))
$$

for all $m \in \mathbf{Z}_{+}$and $\xi \in T^{*}(M)$, where $\tau^{T^{*}(M)}: T^{*}(M) \times M \rightarrow T^{*}(M)$ is a connection-induced local transport of $T^{*}(M)$ (which is defined in the manner of Definition 2.3) and therefore $a\left(\tau^{T^{*}(M)}(\xi, \cdot)\right) \in \Gamma^{\infty}(\operatorname{Hom}(E, F))$.

The next proposition is an immediate consequence of this definition and will be useful in the following section.

PROPOSITION 3.3. Let $a_{r}(\xi)=\left(A_{r}(p(\xi))\right)\left(\bigotimes^{l-r}(i \xi)\right)$ be the term of homogeneity degree $l-r$ in $\xi \in T^{*}(M)$ in the intrinsic symbol $a=\sum_{r=0}^{l} a_{r}$ of a differential operator $A=\sum_{r=0}^{l} A_{r} \hat{\nabla}^{l-r}$ of order $l \geq 0$, where

$$
A_{r} \in \Gamma^{\infty}\left(\operatorname{Hom}(E, F) \otimes S\left(\bigotimes^{l-r}(T(M))\right)\right) .
$$

Then

$$
\left(D^{m} a_{r}\right)(\xi)= \begin{cases}\frac{i^{l-r}(l-r) !}{(l-r-m) !}\left(A_{r}(p(\xi))\right)\left(\bigotimes^{l-r-m} \xi\right) & \text { if } m \leq l-r \\ 0 & \text { if } m>l-r\end{cases}
$$

and

$$
\left(\hat{\nabla}^{m} a_{r}\right)(\xi)=\left(\left(\hat{\nabla}^{m} A_{r}\right)(p(\xi))\right)\left(\bigotimes^{l-r}(i \xi)\right)
$$

for all $m \in \mathbf{Z}_{+}$and $\xi \in T^{*}(M)$. Furthermore, $\hat{\nabla}^{n} D^{m} a_{r}=D^{m} \hat{\nabla}^{n} a_{r}$ for all $n, m \in \mathbf{Z}_{+}$.

REMARK 3.5. Definition 3.2 was given in [27]. As shown there, it is equivalent to the induced connection on the induced homomorphism bundle $p^{*} \operatorname{Hom}(E, F)$ with base $T^{*}(M)$ and is therefore quite natural. It differs from that introduced by Widom $[\mathbf{4 6}, \mathbf{4 7}]$ in the way that $\hat{\nabla}^{m} a$ is defined, it enjoys certain calculational advantages, such as (3.4), which reduce the labor involved in applications of the symbolic calculus.

The formula for the intrinsic symbol of a product of two pseudodifferential operators is fundamental to the symbolic calculus and to the results of the next section. We state it as the following proposition, in which $G$ is a $C^{\infty}$ vector bundle with base $M$. 
Proposition 3.4. Let $A \in L^{l_{1}}(M, E, F)$ and $B \in L^{l_{2}}(M, F, G)$ be two pseudodifferential operators, one of which is properly supported, and let

$$
a \in S^{l_{1}}\left(T^{*}(M), \operatorname{Hom}(E, F)\right) \quad \text { and } \quad b \in S^{l_{2}}\left(T^{*}(M), \operatorname{Hom}(F, G)\right)
$$

be their respective intrinsic symbols. The intrinsic symbol

$$
c \in S^{l_{1}+l_{2}}\left(T^{*}(M), \operatorname{Hom}(E, G)\right)
$$

of the product $C=B A$ may be written $c \sim \sum_{u \geq 0} P_{u}(b, a)$, where $P_{u}(b, a) \in$ $S^{l_{1}+l_{2}-u}\left(T^{*}(M), \operatorname{Hom}(E, G)\right)$ for all $u \in \mathbf{Z}_{+}$and is given explicitly by

$$
\begin{aligned}
P_{u}(b, a)= & \sum_{\bar{n} \geq 0, k \geq 0, n_{\kappa} \geq 0, m_{\kappa} \geq 0}^{\bar{n}+k+|n|+|m|=u} i^{-u}\left(\bar{n} ! k ! n_{+} ! m_{+} !\right)^{-1} \\
& \times\left(D^{N} b\right)\left(\hat{\nabla}^{\bar{n}} D^{M} a\right)\left(\hat{\nabla}^{n_{0}} \hat{\nabla}^{m_{0}} \tau^{E}\right)\left(\bigotimes_{\kappa=1}^{k} \hat{\nabla}^{1+n_{\kappa}} \hat{\nabla}^{1+m_{\kappa}} \phi\right) .
\end{aligned}
$$

In (3.5), $n=\left(n_{0}, n_{1}, \ldots, n_{k}\right) \in \mathbf{Z}_{+}^{k+1}$ and $m=\left(m_{0}, m_{1}, \ldots, m_{k}\right) \in \mathbf{Z}_{+}^{k+1}$ are multi-indices,

$$
\begin{aligned}
& n_{+} !=n_{0} ! \prod_{\kappa=1}^{k}\left(1+n_{\kappa}\right) !, \quad N=\bar{n}+k+|n|, \\
& m_{+} !=m_{0} ! \prod_{\kappa=1}^{k}\left(1+m_{\kappa}\right) !, \quad M=k+|m|,
\end{aligned}
$$

and the various factors are contracted as follows: The (inner) covariant derivatives of the form $\hat{\nabla}^{m_{0}}$ and $\hat{\nabla}^{1+m_{\kappa}}$ are contracted with the $D^{M}$; the (outer) covariant derivatives of the form $\hat{\nabla}^{\bar{n}}, \hat{\nabla}^{n_{0}}$, and $\hat{\nabla}^{1+n_{\kappa}}$ are contracted with the $D^{N}$.

REMARK 3.6. In (3.5) and in the remainder of this paper we adopt certain notational abbreviations which require some explanation:

(i) For all $r, s \in \mathbf{Z}_{+}, \hat{\nabla}^{r} \hat{\nabla}^{s} \phi: T^{*}(M) \rightarrow S\left(\bigotimes^{s}\left(T^{*}(M)\right)\right) \otimes S\left(\bigotimes^{r}\left(T^{*}(M)\right)\right)$ is the $C^{\infty}$ mapping defined by

$$
\left(\hat{\nabla}^{r} \hat{\nabla}^{s} \phi\right)(\xi)=\left(\hat{\nabla}^{r} \hat{\nabla}^{s} \phi(\xi, \cdot)\right)(p(\xi))
$$

for all $\xi \in T^{*}(M)$.

For all $r, s \in \mathbf{Z}_{+}, \hat{\nabla}^{r} \hat{\nabla}^{s} \tau^{E} \in \Gamma^{\infty}\left(\operatorname{End}(E) \otimes S\left(\bigotimes^{s}\left(T^{*}(M)\right)\right) \otimes S\left(\bigotimes^{r}\left(T^{*}(M)\right)\right)\right)$ is defined by

$$
\left(\left(\hat{\nabla}^{r} \hat{\nabla}^{s} \tau^{E}\right)(x)\right)(e)=\left(\hat{\nabla}^{r} \hat{\nabla}^{s} \tau^{E}(e, \cdot)\right)(x)
$$

for all $x \in M$ and $e \in E_{x}$.

For all $r, s \in \mathbf{Z}_{+}, \hat{\nabla}^{r} \hat{\nabla}^{s} \Phi \in \Gamma^{\infty}\left(T(M) \otimes S\left(\bigotimes^{s}\left(T^{*}(M)\right)\right) \otimes S\left(\bigotimes^{r}\left(T^{*}(M)\right)\right)\right)$ is defined by

$$
\left(\left(\hat{\nabla}^{r} \hat{\nabla}^{s} \Phi\right)(x)\right)(\xi)=\left(\hat{\nabla}^{r} \hat{\nabla}^{s} \phi(\xi, \cdot)\right)(x)
$$

for all $x \in M$ and $\xi \in T_{x}^{*}(M)$.

$\hat{\nabla}^{r} \hat{\nabla}^{s} \tau^{E}$ and $\hat{\nabla}^{r} \hat{\nabla}^{s} \Phi$ will often have an implicit right composition with $p$ understood; for example, in (3.5) we really mean $\left(\hat{\nabla}^{n_{0}} \hat{\nabla}^{m_{0}} \tau^{E}\right) \circ p$. 
(ii) We shall write

$$
\left(\bigotimes_{\kappa=1}^{k} W_{\kappa}\right)=W_{1} \otimes W_{2} \otimes \cdots \otimes W_{k}
$$

for the tensor product,

$$
\left(\bigotimes_{\kappa=1}^{k} W_{\kappa}\right)=W_{k} \otimes \cdots \otimes W_{2} \otimes W_{1}
$$

for the reversed tensor product, and shall often suppress $\otimes$ itself; for example, in (3.5) we really mean

$$
\left(D^{N} b\right) \otimes\left(\hat{\nabla}^{\bar{n}} D^{M} a\right) \otimes\left(\hat{\nabla}^{n_{0}} \hat{\nabla}^{m_{0}} \tau^{E}\right) \otimes\left(\bigotimes_{\kappa=1}^{k} \hat{\nabla}^{1+n_{\kappa}} \hat{\nabla}^{1+m_{\kappa}} \phi\right) .
$$

(iii) Tensor contractions will be described verbally, as in Proposition 3.4. In view of their later complexity, it is easier to do so than to introduce an unambiguous, concise notation. Fiber contractions between the homomorphism-valued objects appearing in (3.5) and elsewhere will not be described at all, it being understood that the usual operator (or matrix) product is meant.

(iv) Summations over subscripted quantities are meant to extend to all values of the subscript(s). Likewise, summation constraints which involve subscripted quantities are meant to extend to all values of the subscript(s), unless explicitly indicated otherwise.

REMARK 3.7. The expression (3.5) for $P_{u}(b, a)$ differs slightly from the corresponding formulas of Widom [46, 47] and Drager [16], and the difference is due to our Definition 3.2 of covariant differentiation of an intrinsic symbol. Proposition 3.4 was proved in [27].

REMARK 3.8. It is clear from (3.5) that $P_{u}$ is bilinear and that $P_{0}(b, a)=b a$. Furthermore, if $u \geq 1$ then $P_{u}(b, a)$ consists only of terms which involve at least one derivative of both $b$ and $a$. (If this is not obvious, observe that $N=0$ only if $\bar{n}=k=n_{0}=0$, and the constraint $\bar{n}+k+|n|+|m|=u \geq 1$ then implies $m_{0} \geq 1$. Such terms vanish because of (2.2). Likewise, terms with $\bar{n}+M=0$ also vanish.) These facts will be useful in the next section.

\section{The intrinsic symbol of a resolvent parametrix.}

Definition 4.1. Let $A \in L^{l}(M, E, E)$ and $\lambda \in$ C. $B_{\lambda} \in L^{-l}(M, E, E)$ will be called a resolvent parametrix of $A$ if it is a parametrix of $A-\lambda$ (that is, $B_{\lambda}(A-\lambda)$ differs from the identity by at most an operator with $C^{\infty}$ integral kernel).

In this section we shall present an explicit construction of the asymptotic expansion of the intrinsic symbol of a resolvent parametrix of $A$ in terms of covariant derivatives of $\phi, \tau^{E}$, and the terms in the asymptotic expansion of the intrinsic symbol of $A$. This is the heart of the paper and contains our main results, Theorems 4.1 and 4.2 .

To begin, we need an appropriate ellipticity condition on $A$, which, for simplicity, we assume henceforth to be a classical pseudodifferential operator, although in much of what follows this restriction may be removed. ( $A$ is said to be classical if its intrinsic symbol has an asymptotic expansion $a \sim \sum_{r \geq 0} a_{r}$ such that 
$a_{r} \in S^{l-r}\left(T^{*}(M) \backslash 0, \operatorname{End}(E)\right)$ and $a_{r}$ is positive-homogeneous of degree $l-r$ in $\xi$ for all $r \in \mathbf{Z}_{+}$.)

DEFINITION 4.2. Let $A \in L^{l}(M, E, E)$ be classical with positive-homogeneous principal symbol $a_{0}$ and let $\Gamma=\{\lambda \in \mathbf{C}: \arg \lambda=\theta\}$ be a ray in $\mathbf{C}$. $A$ will be called elliptic with respect to $\Gamma$ if $a_{0}(\xi)-\lambda: E_{p(\xi)} \rightarrow E_{p(\xi)}$ is injective for all $\xi \in T^{*}(M) \backslash 0$ and $\lambda \in \Gamma$.

REMARK 4.1. The terminology used here is similar to that of Gilkey and Smith [21]. Such a $\Gamma$ is called a ray of minimal growth (of the resolvent) [39]. It is necessary to consider $\lambda$ on such a ray to ensure that the construction below results in a resolvent parametrix which remains valid as $|\lambda| \rightarrow \infty$.

We shall construct the intrinsic symbol of a resolvent parametrix of $A$ by using the $P_{u}$ operation of Proposition 3.4:

Proposition 4.1. Let $A \in L^{l}(M, E, E)$ be classical and elliptic with respect to a ray $\Gamma$ in $\mathbf{C}$. If $\lambda \in \Gamma$ then there exists a resolvent parametrix $B_{\lambda} \in L^{-l}(M, E, E)$ of $A$. Furthermore, if the intrinsic symbol of $A$ has classical asymptotic expansion a $\sum_{r \geq 0} a_{r}$, where $a_{r} \in S^{l-r}\left(T^{*}(M) \backslash 0, \operatorname{End}(E)\right)$ is positive-homogeneous of degree $l-r$ for all $r \in \mathbf{Z}_{+}$, then the intrinsic symbol of $B_{\lambda}$ has asymptotic expansion $b \sim \sum_{s \geq 0} b_{s}$, where $b_{s} \in S^{-l-s}\left(T^{*}(M) \backslash 0, \operatorname{End}(E)\right)$ is positive-homogeneous of degree $-l-s$ in $\left(\xi, \lambda^{1 / l}\right)$ for all $\xi \in T^{*}(M) \backslash 0, \lambda \in \Gamma$, and $s \in \mathbf{Z}_{+}$, and is given explicitly by

$$
b_{0}=\left(a_{0}-\lambda\right)^{-1}
$$

and

$$
b_{s}=-\sum_{\substack{r \geq 0, u \geq 0, q \geq 0 \\ r+u \geq 1}}^{r+u+q=s} P_{u}\left(b_{q}, a_{r}\right) b_{0} \quad \text { for all } s \geq 1 .
$$

PROOF. Let $\left\{b_{s}\right\}$ be defined by the requirement

$$
\sum_{r \geq 0, u \geq 0, q \geq 0}^{r+u+q \leq s} P_{u}\left(b_{q}, \tilde{a}_{r}\right)=I_{E} \quad \text { for all } s \in \mathbf{Z}_{+},
$$

where $I_{E}$ is the identity endomorphism of $E$ and $\tilde{a}_{r}$ is defined for all $r \in \mathbf{Z}_{+}$by

$$
\tilde{a}_{r}= \begin{cases}a_{0}-\lambda & \text { if } r=0 \\ a_{r} & \text { if } r \geq 1\end{cases}
$$

(4.3) is equivalent to

$$
P_{0}\left(b_{0}, \tilde{a}_{0}\right)=I_{E}
$$

and

$$
\sum_{r \geq 0, u \geq 0, q \geq 0}^{r+u+q=s} P_{u}\left(b_{q}, \tilde{a}_{r}\right)=0 \text { for all } s \geq 1 .
$$

Since $P_{0}(b, a)=b a,(4.4)$ immediately gives (4.1). Isolating the $q=s$ term in (4.5) and multiplying on the right by $b_{0}$ gives

$$
b_{s}=-\sum_{\substack{r \geq 0, u \geq 0, q \geq 0 \\ r+u \geq 1}}^{r+u+q=s} P_{u}\left(b_{q}, \tilde{a}_{r}\right) b_{0} \quad \text { for all } s \geq 1 .
$$


To obtain (4.2) we must justify the replacement of $\tilde{a}_{r}$ by $a_{r}$ in (4.6). For those terms with $r \geq 1$ this is trivial. For those terms with $r=0$ we know that $u \geq 1$ and hence can invoke Remark 3.8: $\tilde{a}_{0}$ is always differentiated and so may be replaced by $a_{0}$.

To verify that $b_{s} \in S^{-l-s}\left(T^{*}(M) \backslash 0\right.$, End $\left.(E)\right)$ for all $s \in \mathbf{Z}_{+}$, first observe that (4.1) establishes this result for $s=0$. Next, assume that

$$
b_{q} \in S^{-l-q}\left(T^{*}(M) \backslash 0, \operatorname{End}(E)\right) \text { for all } 0 \leq q \leq s-1 .
$$

Since $P_{u}: S^{l_{2}} \times S^{l_{1}} \rightarrow S^{l_{1}+l_{2}-u}$, it follows from (4.2) that

$$
b_{s} \in S^{-l-s}\left(T^{*}(M) \backslash 0, \operatorname{End}(E)\right),
$$

and so the result is proved by induction.

A similar inductive proof establishes that $b_{s}$ is positive-homogeneous of degree $-l-s$ in $\left(\xi, \lambda^{1 / l}\right)$ for all $\xi \in T^{*}(M) \backslash 0, \lambda \in \Gamma$, and $s \in \mathbf{Z}_{+}$.

The statement $b \sim \sum_{s \geq 0} b_{s}$ now defines $b$ modulo $S^{-\infty}\left(T^{*}(M) \backslash 0\right.$, End $\left.(E)\right)$, and it follows from (4.3) that $\sum_{u \geq 0} P_{u}(b, a-\lambda) \sim I_{E}$. The operator $B_{\lambda}$ with intrinsic symbol $b$, which may be obtained (modulo smoothing) from Proposition 3.2, is, therefore, a parametrix of $A-\lambda$. (Elements of $S^{-\infty}$ map to smoothing operators under (3.3).) This establishes existence.

REMARK 4.2. Although the above construction regards $B_{\lambda}$ as a left parametrix of $A-\lambda$, it is also a right parametrix. Indeed, an alternative procedure is to define $\left\{b_{s}\right\}$ by the requirement

$$
\sum_{r \geq 0, u \geq 0, q \geq 0}^{r+u+q \leq s} P_{u}\left(\tilde{a}_{r}, b_{q}\right)=I_{E} \quad \text { for all } s \in \mathbf{Z}_{+},
$$

and thereby obtain, instead of $(4.2)$,

$$
b_{s}=-\sum_{\substack{r \geq 0, u \geq 0, q \geq 0 \\ r+u \geq 1}}^{r+u+q=s} b_{0} P_{u}\left(a_{r}, b_{q}\right) \quad \text { for all } s \geq 1 .
$$

However, because in the explicit formula (3.5) for $P_{u}$ the only derivatives on the first argument are along the fiber, we shall find (4.2) the more convenient choice in what follows. (It should be noted that (4.2) and (4.7) do not yield identical expressions for $b_{s}$, but rather expressions which are equivalent upon application of the Ricci identities.)

REMARK 4.3. It is possible to dispense with the requirement that $a$ be expanded as an asymptotic series (in particular, with the requirement that $A$ be classical) and still obtain a recursion relation for the terms in an asymptotic series for $b$. This is the approach taken by Widom $[\mathbf{4 6}, \mathbf{4 7}]$; his results may be reproduced, in part, from Proposition 4.1 and Remark 4.2 by setting $a_{0}=a$ and $a_{r}=0$ for all $r \geq 1$, so only the $r=0$ terms survive in (4.7) (or (4.2)).

PROPOSITION 4.2. The solution of the recursion relation (4.2) is the nested expression

$$
b_{s}=\sum_{J=1}^{s}(-1)^{J} \sum_{\substack{r_{j} \geq 0, u_{j} \geq 0 \\ r_{j}+u_{j} \geq 1}}^{|r+u|=s} P_{u_{1}}\left(P_{u_{2}}\left(\cdots P_{u_{J}}\left(b_{0}, a_{r_{J}}\right) b_{0}, \ldots, a_{r_{2}}\right) b_{0}, a_{r_{1}}\right) b_{0}
$$

where $r=\left(r_{1}, \ldots, r_{J}\right) \in \mathbf{Z}_{+}^{J}$ and $u=\left(u_{1}, \ldots, u_{J}\right) \in \mathbf{Z}_{+}^{J}$ are multi-indices. 
ProOF. From (4.2), if $s \geq 1$ then

$$
b_{s}=-\sum_{\substack{r_{1} \geq 0, u_{1} \geq 0 \\ r_{1}+u_{1} \geq 1}}^{r_{1}+u_{1}=s} P_{u_{1}}\left(b_{0}, a_{r_{1}}\right) b_{0}-\sum_{\substack{r_{1} \geq 0, u_{1} \geq 0, q_{1} \geq 0 \\ r_{1}+u_{1} \geq 1}}^{r_{1}+u_{1}+q_{1}=s} P_{u_{1}}\left(b_{q_{1}}, a_{r_{1}}\right) b_{0} .
$$

Since $q_{1} \geq 1$ in the second summation, we may substitute a similar expression for $b_{q_{1}}$ to obtain

$$
\begin{aligned}
& b_{s}=-\sum_{\substack{r_{1} \geq 0, u_{1} \geq 0 \\
r_{1}+u_{1} \geq 1}}^{r_{1}+u_{1}=s} P_{u_{1}}\left(b_{0}, a_{r_{1}}\right) b_{0} \\
& -\sum_{r_{1} \geq 0, u_{1} \geq 0, q_{1} \geq 1}^{r_{1}+u_{1}+q_{1}=s} P_{u_{1}}\left(-\sum_{\substack{r_{2} \geq 0, u_{2} \geq 0 \\
r_{2}+u_{2} \geq 1}}^{r_{2}+u_{2}=q_{1}} P_{u_{2}}\left(b_{0}, a_{r_{2}}\right) b_{0}\right. \\
& \left.-\sum_{r_{2} \geq 0, u_{2} \geq 0, q_{2} \geq 1}^{r_{2}+u_{2}+q_{2}=q_{1}} P_{u_{2}}\left(b_{q_{2}}, a_{r_{2}}\right) b_{0}, a_{r_{1}}\right) b_{0} \\
& =-\sum_{\substack{r_{1} \geq 0, u_{1} \geq 0 \\
r_{1}+u_{1} \geq 1}}^{r_{1}+u_{1}=s} P_{u_{1}}\left(b_{0}, a_{r_{1}}\right) b_{0}+\sum_{\substack{r_{1} \geq 0, u_{1} \geq 0, r_{2} \geq 0, u_{2} \geq 0 \\
r_{1}+u_{1} \geq 1, r_{2}+u_{2} \geq 1}}^{r_{1}+u_{1}+r_{2}+u_{2}=s} P_{u_{1}}\left(P_{u_{2}}\left(b_{0}, a_{r_{2}}\right) b_{0}, a_{r_{1}}\right) b_{0} \\
& +\sum_{\substack{r_{1} \geq 0, u_{1} \geq 0, r_{2} \geq 0, u_{2} \geq 0, q_{2} \geq 1 \\
r_{1}+u_{1} \geq 1, r_{2}+u_{2} \geq 1}}^{r_{1}+u_{1}+r_{2}+u_{2}+q_{2}=s} P_{u_{1}}\left(P_{u_{2}}\left(b_{q_{2}}, a_{r_{2}}\right) b_{0}, a_{r_{1}}\right) b_{0}
\end{aligned}
$$

where we have used the linearity of $P_{u_{1}}$ in its first argument and have simplified the summations. Continuing in this way leads to

$$
\begin{aligned}
b_{2}= & -\sum_{\substack{r_{1} \geq 0, u_{1} \geq 0 \\
r_{1}+u_{1} \geq 1}}^{r_{1}+u_{1}=s} P_{u_{1}}\left(b_{0}, a_{r_{1}}\right) b_{0}+\sum_{\substack{r_{1} \geq 0, u_{1} \geq 0, r_{2} \geq 0, u_{2} \geq 0 \\
r_{1}+u_{1} \geq 1, r_{2}+u_{2} \geq 1}}^{r_{1}+u_{1}+r_{2}+u_{2}=s} P_{u_{1}}\left(P_{u_{2}}\left(b_{0}, a_{r_{2}}\right) b_{0}, a_{r_{1}}\right) b_{0} \\
& +\cdots+(-1)^{s} \sum_{\substack{r_{1}+u_{1}+\cdots+r_{s}+u_{s}=s \\
r_{1} \geq 0, u_{1} \geq 0, \ldots, r_{s} \geq 0, u_{s} \geq 0 \\
r_{1}+u_{1} \geq 1, \ldots, r_{s}+u_{s} \geq 1}}^{r_{u_{1}}\left(\cdots P_{u_{s}}\left(b_{0}, a_{r_{s}}\right) b_{0}, \ldots, a_{r_{1}}\right) b_{0}} \\
& +(-1)^{s} \sum_{\substack{r_{1}+u_{1}+\cdots+r_{s}+u_{s}+q_{s}=s \\
r_{1} \geq 0, u_{1} \geq 0, \ldots, r_{s} \geq 0, u_{s} \geq 0, q_{s} \geq 1 \\
r_{1}+u_{1} \geq 1, \ldots, r_{s}+u_{s} \geq 1}} P_{u_{1}}\left(\cdots P_{u_{s}}\left(b_{q_{s}}, a_{r_{s}}\right) b_{0}, \ldots, a_{r_{1}}\right) b_{0} .
\end{aligned}
$$

However, the last summation is vacuous, since the lower bounds imply $r_{1}+u_{1}+$ $\cdots+r_{s}+u_{s}+q_{s} \geq s+1$. Hence the series of nests terminates, and (4.8) results.

A more explicit expression for $b_{s}$ may be obtained by using (3.5) in (4.8). This will be done in Proposition 4.3, but first we identify some quantities which appear there and beyond. 
DEFINITION 4.3. In the remainder of this paper,

$$
\begin{aligned}
& r=\left(r_{1}, \ldots, r_{J}\right) \in \mathbf{Z}_{+}^{J}, \\
& \bar{n}=\left(\bar{n}_{1}, \ldots, \bar{n}_{J}\right) \in \mathbf{Z}_{+}^{J} \text {, } \\
& k=\left(k_{1}, \ldots, k_{J}\right) \in \mathbf{Z}_{+}^{J}, \\
& n_{j}=\left(n_{j 0}, \ldots, n_{j k_{j}}\right) \in \mathbf{Z}_{+}^{k_{j}+1} \quad \text { for all } 1 \leq j \leq J, \\
& m_{j}=\left(m_{j 0}, \ldots, m_{j k_{j}}\right) \in \mathbf{Z}_{+}^{k_{j}+1} \quad \text { for all } 1 \leq j \leq J, \\
& n=\left(n_{j \kappa}\right) \\
& m=\left(m_{j \kappa}\right) \\
& |n|=\sum_{j=1}^{J}\left|n_{j}\right|=\sum_{j=1}^{J} \sum_{\kappa=0}^{k_{j}} n_{j \kappa}, \\
& |m|=\sum_{j=1}^{J}\left|m_{j}\right|=\sum_{j=1}^{J} \sum_{\kappa=0}^{k_{j}} m_{j \kappa}, \\
& n_{+} !=\prod_{j=1}^{J}\left(n_{j 0} ! \prod_{\kappa=1}^{k_{j}}\left(1+n_{j \kappa}\right) !\right) \\
& m_{+} !=\prod_{j=1}^{J}\left(m_{j 0} ! \prod_{\kappa=1}^{k_{j}}\left(1+m_{j \kappa}\right) !\right) \\
& N_{j}=\bar{n}_{j}+k_{j}+\left|n_{j}\right| \quad \text { for all } 1 \leq j \leq J \text {, and } \\
& M_{j}=k_{j}+\left|m_{j}\right| \quad \text { for all } 1 \leq j \leq J .
\end{aligned}
$$

Proposition 4.3. Let $A \in L^{l}(M, E, E)$ be classical and elliptic with respect to a ray $\Gamma$ in $\mathbf{C}$, let $\lambda \in \Gamma$, and let $B_{\lambda} \in L^{-l}(M, E, E)$ be a resolvent parametrix of $A$. If the intrinsic symbol of $A$ has classical asymptotic expansion $a \sim \sum_{r \geq 0} a_{r}$ then the intrinsic symbol of $B_{\lambda}$ has asymptotic expansion $b \sim \sum_{s \geq 0} b_{s}$, where $b_{0}=\left(a_{0}-\lambda\right)^{-1}$ and, for all $s \geq 1$,

$$
\begin{aligned}
& b_{s}=\sum_{J=1}^{s} \sum_{\substack{r_{j} \geq 0, \bar{n}_{j} \geq 0, k_{j} \geq 0, n_{j \kappa} \geq 0, m_{j \kappa} \geq 0 \\
r_{j}+\bar{n}_{j}+k_{j}+\left|n_{j}\right|+\left|m_{j}\right| \geq 1}}^{|r+\bar{n}+k|+|n+m|=s} i^{2 J+|r|-s}\left(\bar{n} ! k ! n_{+} ! m_{+} !\right)^{-1} \\
& \times D^{N_{1}}\left[\cdots \left[D ^ { N _ { j } } \left[\cdots \left[\left(D^{N_{J}} b_{0}\right)\left(\hat{\nabla}^{\bar{n}_{J}} D^{M_{J}} a_{r_{J}}\right)\right.\right.\right.\right. \\
& \left.\left.\otimes\left(\hat{\nabla}^{n_{J 0}} \hat{\nabla}^{m_{J 0}} \tau^{E}\right)\left(\bigotimes_{\kappa=1}^{k_{J}} \hat{\nabla}^{1+n_{J \kappa}} \hat{\nabla}^{1+m_{J \kappa} \phi}\right) b_{0}\right] \cdots\right] \\
& \left.\left.\otimes\left(\hat{\nabla}^{\bar{n}_{j}} D^{M_{j}} a_{r_{j}}\right)\left(\hat{\nabla}^{n_{j 0}} \hat{\nabla}^{m_{j 0}} \tau^{E}\right)\left(\bigotimes_{\kappa=1}^{k_{j}} \hat{\nabla}^{1+n_{j \kappa}} \hat{\nabla}^{1+m_{j \kappa} \phi}\right) b_{0}\right] \cdots\right] \\
& \otimes\left(\hat{\nabla}^{\bar{n}_{1}} D^{M_{1}} a_{r_{1}}\right)\left(\hat{\nabla}^{n_{10}} \hat{\nabla}^{m_{10}} \tau^{E}\right)\left(\bigotimes_{\kappa=1}^{k_{1}} \hat{\nabla}^{1+n_{1 \kappa}} \hat{\nabla}^{1+m_{1 \kappa}} \phi\right) b_{0} .
\end{aligned}
$$


All subscripted quantities in (4.9) are as listed in Definition 4.3 and the various factors are contracted as follows: The (inner) covariant derivatives of the form $\hat{\nabla}^{m_{j 0}}$ and $\hat{\nabla}^{1+m_{j \kappa}}$ are contracted with the $D^{M_{j}}$ for each $1 \leq j \leq J$; the (outer) covariant derivatives of the form $\hat{\nabla}^{\bar{n}_{j}}, \hat{\nabla}^{n_{j 0}}$, and $\hat{\nabla}^{1+n_{j_{\kappa}}}$ are contracted with the $D^{N_{j}}$ for each $1 \leq j \leq J$.

PrOOF. This is a simple consequence of Propositions 3.4 and 4.2 in the following way. Inserting (3.5) in (4.8) and using the linearity of $P_{u_{j}}$ in its first argument, one encounters the summation

$$
\sum_{\substack{r_{j} \geq 0, u_{j} \geq 0 \\ r_{j}+u_{j} \geq 1}}^{|r+u|=s} \sum_{\bar{n}_{1} \geq 0, k_{1} \geq 0, n_{1 \kappa} \geq 0, m_{1 \kappa} \geq 0}^{\bar{n}_{1}+k_{1}+\left|n_{1}\right|+\left|m_{1}\right|=u_{1}} \cdots \sum_{\bar{n}_{J} \geq 0, k_{J} \geq 0, n_{J \kappa} \geq 0, m_{J \kappa} \geq 0}^{\bar{n}_{J}+k_{J}+\left|n_{J}\right|+\left|m_{J}\right|=u_{J}} .
$$

This is simply a splitting of the summation over all distinct arrangements of $s$ elements,

$$
\sum_{\substack{r_{j} \geq 0, \bar{n}_{j} \geq 0, k_{j} \geq 0, n_{j \kappa} \geq 0, m_{j \kappa} \geq 0 \\ r_{j}+\bar{n}_{j}+k_{j}+\left|n_{j}\right|+\left|m_{j}\right| \geq 1}}^{|r+\bar{n}+k|+|n+m|=s}
$$

into a summation over distinct classes specified by $\left(r_{1}, \ldots, r_{J}\right)$ and $\left(u_{1}, \ldots, u_{J}\right)$, where $u_{j}=\bar{n}_{j}+k_{j}+\left|n_{j}\right|+\left|m_{j}\right|$ for all $1 \leq j \leq J$, followed by summations over distinct arrangements within each class. (4.9) and its accompanying contractions result from this observation, Definition 4.3 , and the contractions specified in Proposition 3.4.

The following lemmas will be useful in dealing with the nested $T^{*}(M)$ fiber derivatives in (4.9). The first is simply a version of the Leibnitz formula and so its proof will be omitted.

LEMMA 4.1. Let $\mathbf{Z} \ni I \geq 1$ and, for all $i \in \mathbf{Z}$ such that $1 \leq i \leq I$, let $H_{i}$ be a $C^{\infty}$ vector bundle with base $M$ and $h_{i}: T^{*}(M) \rightarrow H_{i}$ a $C^{\infty}$ mapping which commutes with base projections. If $D$ denotes differentiation along fibers of $T^{*}(M)$ then

$$
D^{N}\left(\bigotimes_{i=1}^{I} h_{i}\right)=\sum_{q_{i} \geq 0}^{|q|=N} \frac{N !}{q !} S_{N}\left(\bigotimes_{i=1}^{I} D^{q_{i}} h_{i}\right)
$$

for all $N \in \mathbf{Z}_{+}$, where $q=\left(q_{1}, \ldots, q_{I}\right) \in \mathbf{Z}_{+}^{I}$ and $S_{N}$ indicates symmetrization in the $N$ contravariant indices of the $\left\{D^{q_{i}}\right\}$.

LEMMA 4.2. Let $b_{0}=\left(a_{0}-\lambda\right)^{-1}$. Then

$$
D^{N} b_{0}=\sum_{T=1}^{N}(-1)^{T} \sum_{q_{t} \geq 1}^{|q|=N} \frac{N !}{q !} S_{N}\left(\bigotimes_{t=1}^{T} b_{0} D^{q_{t}} a_{0}\right) b_{0}
$$

for all $\mathbf{Z} \ni N \geq 1$, where $q=\left(q_{1}, \ldots, q_{T}\right) \in \mathbf{Z}_{+}^{T}$ and $S_{N}$ indicates symmetrization in the $N$ contravariant indices of the $\left\{D^{q_{t}}\right\}$.

PROOF. We shall present a constructive proof which is similar in spirit to that of Proposition 4.2. (Both results can also be proved by induction.)

Since $N \geq 1$,

$$
D^{N}\left(\left(a_{0}-\lambda\right) b_{0}\right)=0 .
$$


Using Lemma 4.1 on the left-hand side, we obtain

$$
\sum_{q_{1} \geq 0, p_{1} \geq 0}^{q_{1}+p_{1}=N} \frac{N !}{q_{1} ! p_{1} !} S_{N}\left[\left(D^{q_{1}}\left(a_{0}-\lambda\right)\right) \otimes\left(D^{p_{1}} b_{0}\right)\right]=0,
$$

and, on isolating the terms with $q_{1}=0$ or $p_{1}=0$,

$$
\left(a_{0}-\lambda\right)\left(D^{N} b_{0}\right)+\left(D^{N} a_{0}\right) b_{0}+\sum_{q_{1} \geq 1, p_{1} \geq 1}^{q_{1}+p_{1}=N} \frac{N !}{q_{1} ! p_{1} !} S_{N}\left[\left(D^{q_{1}} a_{0}\right) \otimes\left(D^{p_{1}} b_{0}\right)\right]=0 .
$$

Hence, if $N \geq 1$,

$$
D^{N} b_{0}=-b_{0}\left(D^{N} a_{0}\right) b_{0}-\sum_{q_{1} \geq 1, p_{1} \geq 1}^{q_{1}+p_{1}=N} \frac{N !}{q_{1} ! p_{1} !} S_{N}\left[b_{0}\left(D^{q_{1}} a_{0}\right) \otimes\left(D^{p_{1}} b_{0}\right)\right] .
$$

Since $p_{1} \geq 1$ in the summation on the right, we may substitute a similar expression for $D^{p_{1}} b_{0}$ to obtain

$$
\begin{aligned}
D^{N} b_{0}= & -b_{0}\left(D^{N} a_{0}\right) b_{0} \\
& -\sum_{q_{1} \geq 1, p_{1} \geq 1}^{q_{1}+p_{1}=N} \frac{N !}{q_{1} ! p_{1} !} S_{N}\left[b _ { 0 } ( D ^ { q _ { 1 } } a _ { 0 } ) \otimes \left(-b_{0}\left(D^{p_{1}} a_{0}\right) b_{0}\right.\right. \\
& \left.\left.-\sum_{q_{2} \geq 1, p_{2} \geq 1}^{q_{2}+p_{2}=p_{1}} \frac{p_{1} !}{q_{2} ! p_{2} !} b_{0}\left(D^{q_{2}} a_{0}\right) \otimes\left(D^{p_{2}} b_{0}\right)\right)\right] \\
= & -b_{0}\left(D^{N} a_{0}\right) b_{0}+\sum_{q_{1} \geq 1, q_{2} \geq 1}^{q_{1}+q_{2}=N} \frac{N !}{q_{1} ! q_{2} !} S_{N}\left[b_{0}\left(D^{q_{1}} a_{0}\right) \otimes b_{0}\left(D^{q_{2}} a_{0}\right) b_{0}\right] \\
& +\sum_{q_{1} \geq 1, q_{2} \geq 1, p_{2} \geq 1}^{q_{1}+q_{2}+p_{2}=N} \frac{N !}{q_{1} ! q_{2} ! p_{2} !} S_{N}\left[b_{0}\left(D^{q_{1}} a_{0}\right) \otimes b_{0}\left(D^{q_{2}} a_{0}\right) \otimes\left(D^{p_{2}} b_{0}\right)\right],
\end{aligned}
$$

where we have simplified the summations. Continuing in this way leads to

$$
\begin{aligned}
& D^{N} b_{0}=-b_{0}\left(D^{N} a_{0}\right) b_{0}+\sum_{q_{1} \geq 1, q_{2} \geq 1}^{q_{1}+q_{2}=N} \frac{N !}{q_{1} ! q_{2} !} S_{N}\left[b_{0}\left(D^{q_{1}} a_{0}\right) \otimes b_{0}\left(D^{q_{2}} a_{0}\right) b_{0}\right] \\
& +\cdots+(-1)^{N} \sum_{q_{1} \geq 1, \ldots, q_{N} \geq 1}^{q_{1}+\cdots+q_{N}=N} \frac{N !}{q_{1} ! \cdots q_{N} !} S_{N}\left[b_{0}\left(D^{q_{1}} a_{0}\right) \otimes \cdots \otimes b_{0}\left(D^{q_{N}} a_{0}\right) b_{0}\right] \\
& +(-1)^{N} \sum_{q_{1} \geq 1, \ldots, q_{N} \geq 1, p_{N} \geq 1}^{q_{1}+\cdots+q_{N}+p_{N}=N} \frac{N !}{q_{1} ! \cdots q_{N} ! p_{N} !} \\
& \cdot S_{N}\left[b_{0}\left(D^{q_{1}} a_{0}\right) \otimes \cdots \otimes b_{0}\left(D^{q_{N}} a_{0}\right) \otimes\left(D^{p_{N}} b_{0}\right)\right] .
\end{aligned}
$$

However, the last summation is vacuous, since the lower bounds imply $q_{1}+\cdots+$ $q_{N}+p_{N} \geq N+1$, and hence we arrive at (4.10).

REMARK 4.4. In Lemma 4.2 (and Corollary 4.1 below) we may, if we wish, remove the upper limit $N$ on the $T$ summation, since if $T \geq N+1$ then the $q$ summation is vacuous because its lower bounds imply $|q| \geq N+1$. 
COROLlaRY 4.1. Let $b_{0}=\left(a_{0}-\lambda\right)^{-1}$, let $H$ be a $C^{\infty}$ vector bundle with base $M$, and let $h: T^{*}(M) \rightarrow H$ be a $C^{\infty}$ mapping which commutes with base projections. If $D$ denotes differentiation along fibers of $T^{*}(M)$ then

$$
D^{N}\left(h \otimes b_{0}\right)=\sum_{T=0}^{N}(-1)^{T} \sum_{\bar{q} \geq 0, q_{t} \geq 1}^{\bar{q}+|q|=N} \frac{N !}{\bar{q} ! q !} S_{N}\left(\left(D^{\bar{q}} h\right) \otimes\left(\bigotimes_{t=1}^{T} b_{0} D^{q_{t}} a_{0}\right) b_{0}\right)
$$

for all $N \in \mathbf{Z}_{+}$, where $q=\left(q_{1}, \ldots, q_{T}\right) \in \mathbf{Z}_{+}^{T}$ and $S_{N}$ indicates symmetrization in the $N$ contravariant indices of the $D^{\bar{q}}$ and $\left\{D^{q_{t}}\right\}$.

ProOF. By Lemma 4.1,

$$
\begin{aligned}
D^{N}\left(h \otimes b_{0}\right) & =\sum_{\bar{q} \geq 0, p \geq 0}^{\bar{q}+p=N} \frac{N !}{\bar{q} ! p !} S_{N}\left(\left(D^{\bar{q}} h\right) \otimes\left(D^{p} b_{0}\right)\right) \\
& =\left(D^{N} h\right) \otimes b_{0}+\sum_{\bar{q} \geq 0, p \geq 1}^{\bar{q}+p=N} \frac{N !}{\bar{q} ! p !} S_{N}\left(\left(D^{\bar{q}} h\right) \otimes\left(D^{p} b_{0}\right)\right) .
\end{aligned}
$$

Since $p \geq 1$ in the summation on the right, we may use Lemma 4.2 to obtain

$$
\begin{aligned}
D^{N}\left(h \otimes b_{0}\right)= & \left(D^{N} h\right) \otimes b_{0} \\
& +\sum_{\bar{q} \geq 0, p \geq 1}^{\bar{q}+p=N} \sum_{T=1}^{p}(-1)^{T} \sum_{q_{t} \geq 1}^{|q|=p} \frac{N !}{\bar{q} ! q !} S_{N}\left(\left(D^{\bar{q}} h\right) \otimes\left(\bigotimes_{t=1}^{T} b_{0} D^{q_{t}} a_{0}\right) b_{0}\right) \\
= & \left(D^{N} h\right) \otimes b_{0} \\
& +\sum_{T \geq 1}(-1)^{T} \sum_{\bar{q} \geq 0, q_{t} \geq 1}^{\bar{q}+|q|=N} \frac{N !}{\bar{q} ! q !} S_{N}\left(\left(D^{\bar{q}} h\right) \otimes\left(\bigotimes_{t=1}^{T} b_{0} D^{q_{t}} a_{0}\right) b_{0}\right),
\end{aligned}
$$

where we have taken advantage of Remark 4.4 in pulling the $T$ summation to the outside and have simplified the remaining summations. If $T \geq N+1$ then the summation on the right is vacuous, since the lower bounds imply $\bar{q}+|q| \geq N+1$; we may therefore insert $N$ as an upper limit on the $T$ summation. (4.11) now follows from the observation that the first term on the right may be included in the summation as the term with $T=0$.

We are now prepared to handle the nested $T^{*}(M)$ fiber derivatives in (4.9), but first we identify some additional quantities which appear below.

DEFINITION 4.4. In the remainder of this paper,

$$
\begin{aligned}
& N=\left(N_{1}, \ldots, N_{J}\right) \in \mathbf{Z}_{+}^{J}, \\
& T=\left(T_{1}, \ldots, T_{J}\right) \in \mathbf{Z}_{+}^{J}, \\
& \bar{p}=\left(\bar{p}_{1}, \ldots, \bar{p}_{J}\right) \in \mathbf{Z}_{+}^{J}, \\
& p=\left(p_{1}, \ldots, p_{J}\right) \in \mathbf{Z}_{+}^{J},
\end{aligned}
$$




$$
\begin{array}{rlrl}
q_{j} & =\left(q_{j 1}, \ldots, q_{j T_{j}}\right) \in \mathbf{Z}_{+}^{T_{j}} & & \text { for all } 1 \leq j \leq J, \\
q & =\left(q_{j t}\right) & & \text { where } 1 \leq j \leq J \text { and } 1 \leq t \leq T_{j}, \\
|q| & =\sum_{j=1}^{J}\left|q_{j}\right|=\sum_{j=1}^{J} \sum_{t=1}^{T_{j}} q_{j t}, & \\
q ! & =\prod_{j=1}^{J}\left(q_{j} !\right)=\prod_{j=1}^{J} \prod_{t=1}^{T_{j}}\left(q_{j t} !\right), & \\
\bar{N}_{j} & =N_{j}+\sum_{i=1}^{j-1}\left(N_{i}-\bar{p}_{i+1}-p_{i+1}-\left|q_{i}\right|\right) & \text { for all } 1 \leq j \leq J, \text { and } \\
\bar{N} & =\left(\bar{N}_{1}, \ldots, \bar{N}_{J}\right) \in \mathbf{Z}_{+}^{J} . &
\end{array}
$$

THEOREM 4.1. Let $A \in L^{l}(M, E, E)$ be classical and elliptic with respect to a ray $\Gamma$ in $\mathbf{C}$, let $\lambda \in \Gamma$, and let $B_{\lambda} \in L^{-l}(M, E, E)$ be a resolvent parametrix of $A$. If the intrinsic symbol of $A$ has classical asymptotic expansion $a \sim \sum_{r \geq 0} a_{r}$ then the intrinsic symbol of $B_{\lambda}$ has asymptotic expansion $b \sim \sum_{s \geq 0} b_{s}$, where $b_{0}=\left(a_{0}-\lambda\right)^{-1}$ and, for all $s \geq 1$,

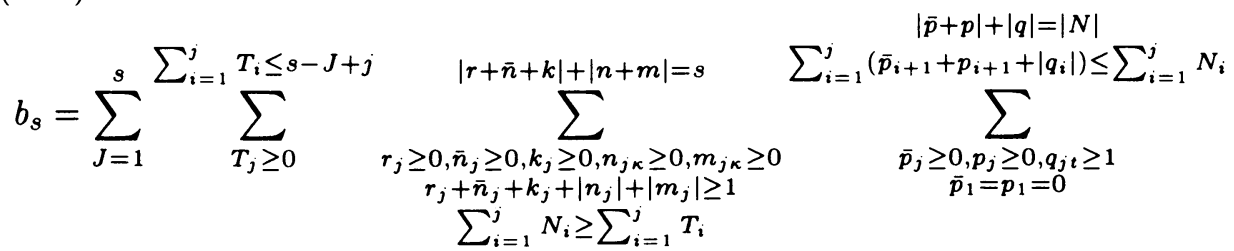

$$
\begin{aligned}
& i^{2(J+|T|)+|r|-s} \bar{N} !\left(\bar{n} ! k ! n_{+} ! m_{+} ! \bar{p} ! p ! q !(\bar{N}-N) !\right)^{-1} \\
& \times\left[\bigotimes _ { j = 1 } ^ { J } R \left[\left(\bigotimes_{t=1}^{T_{j}} b_{0} D^{q_{j t}} a_{0}\right) b_{0}\left(D^{\bar{p}_{j}} \hat{\nabla}^{\bar{n}_{j}} D^{M_{j}} a_{r_{j}}\right)\left(\hat{\nabla}^{n_{j 0}} \hat{\nabla}^{m_{j 0}} \tau^{E}\right)\right.\right. \\
& \left.\left.\otimes\left(D^{p_{j}}\left(\bigotimes_{\kappa=1}^{k_{j}} \hat{\nabla}^{1+n_{j \kappa}} \hat{\nabla}^{1+m_{j \kappa} \phi}\right)\right)\right]\right] b_{0} .
\end{aligned}
$$

All subscripted quantities in (4.12) are as listed in Definitions 4.3 and 4.4. The (inner) covariant derivatives of the form $\hat{\nabla}^{m_{j 0}}$ and $\hat{\nabla}^{1+m_{j \kappa}}$ are contracted with the $D^{M_{j}}$ for each $1 \leq j \leq J$. The (outer) covariant derivatives of the form $\hat{\nabla}^{\bar{n}_{j}}$, $\hat{\nabla}^{n_{j 0}}$, and $\hat{\nabla}^{1+n_{j \kappa}}$ have much more complicated contractions, which are described inductively as follows: For each $1 \leq j \leq J-1$, the $N_{j}$ covariant derivatives of the form $\hat{\nabla}^{\bar{n}_{j}}, \hat{\nabla}^{n_{j 0}}$, and $\hat{\nabla}^{1+n_{j \kappa}}$ are combined with the $\sum_{i=1}^{j-1}\left(N_{i}-\bar{p}_{i+1}-p_{i+1}-\left|q_{i}\right|\right)$ as yet uncontracted covariant derivatives of the form $\hat{\nabla}^{\bar{n}_{i}}, \hat{\nabla}^{n_{i 0}}$, and $\hat{\nabla}^{1+n_{i \kappa}}$ for all $1 \leq i \leq j-1$, and the total of

$$
\bar{N}_{j}=N_{j}+\sum_{i=1}^{j-1}\left(N_{i}-\bar{p}_{i+1}-p_{i+1}-\left|q_{i}\right|\right)
$$

covariant derivatives is symmetrized. Of these, $\left|q_{j}\right|$ are contracted with the $D^{q_{j t}}$, $p_{j+1}$ are contracted with the $D^{p_{j+1}}$, and $\bar{p}_{j+1}$ are contracted with the $D^{\bar{p}_{j+1}}$. The 
remaining

$$
\bar{N}_{j}-\bar{p}_{j+1}-p_{j+1}-\left|q_{j}\right|=\sum_{i=1}^{j}\left(N_{i}-\bar{p}_{i+1}-p_{i+1}-\left|q_{i}\right|\right)
$$

are combined with the $N_{j+1}$ covariant derivatives of the form $\hat{\nabla}^{\bar{n}_{j+1}}, \hat{\nabla}^{n_{j+10}}$, and $\hat{\nabla}^{1+n_{j+1 \kappa}}$, and the process is repeated. After the final such operation (when $j=$ $J-1$ ), the remaining

$$
\bar{N}_{J-1}-\bar{p}_{J}-p_{J}-\left|q_{J-1}\right|=\sum_{i=1}^{J-1}\left(N_{i}-\bar{p}_{i+1}-p_{i+1}-\left|q_{i}\right|\right)
$$

covariant derivatives are combined with the $N_{J}$ covariant derivatives of the form $\hat{\nabla}^{\bar{n}_{J}}, \hat{\nabla}^{n_{J 0}}$, and $\hat{\nabla}^{1+n_{J \kappa}}$, and the total of

$$
\bar{N}_{J}=N_{J}+\sum_{i=1}^{J-1}\left(N_{i}-\bar{p}_{i+1}-p_{i+1}-\left|q_{i}\right|\right)=\left|q_{J}\right|
$$

covariant derivatives is symmetrized and contracted with the $D^{q_{J t}}$.

PROOF. Our intention is to evaluate the nested $T^{*}(M)$ fiber derivatives in (4.9) from the outside in. At the $j$ th step we will encounter an expression of the following form (for some $\bar{N}_{j} \in \mathbf{Z}_{+}$to be determined):

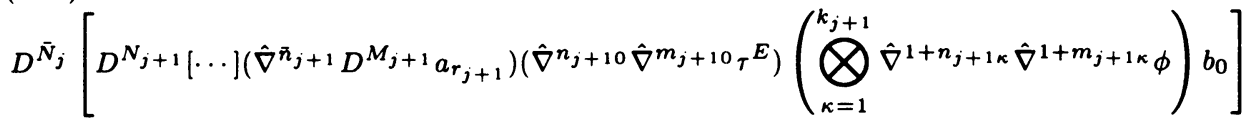

$$
\begin{aligned}
& =\sum_{T_{j}=0}^{\bar{N}_{j}}(-1)^{T_{j}} \sum_{\bar{q}_{j} \geq 0, q_{j t} \geq 1}^{\bar{q}_{j}+\left|q_{j}\right|=\bar{N}_{j}} \frac{\bar{N}_{j} !}{\bar{q}_{j} ! q_{j} !} \sum_{\bar{p}_{j+1} \geq 0, p_{j+1} \geq 0, s_{j+1} \geq 0}^{\bar{p}_{j+1}+p_{j+1}+s_{j+1}=\bar{q}_{j}} \frac{\bar{q}_{j} !}{\bar{p}_{j+1} ! p_{j+1} ! s_{j+1} !} \\
& \times S_{\bar{N}_{j}}\left[D^{N_{j+1}+s_{j+1}}[\cdots]\left(D^{\bar{p}_{j+1}} \hat{\nabla}^{\bar{n}_{j+1}} D^{M_{j+1}} a_{r_{j+1}}\right)\left(\hat{\nabla}^{n_{j+10}} \hat{\nabla}^{m_{j+10}} \tau^{E}\right)\right. \\
& \left.\otimes\left(D^{p_{j+1}}\left(\bigotimes_{\kappa=1}^{k_{j+1}} \hat{\nabla}^{1+n_{j+1} \kappa} \hat{\nabla}^{1+m_{j+1 \kappa} \phi}\right)\right) b_{0}\left(D^{q_{j 1}} a_{0}\right) \cdots b_{0}\left(D^{q_{j} T_{j}} a_{0}\right) b_{0}\right] \\
& =\sum_{T_{j}=0}^{\bar{N}_{j}}(-1)^{T_{j}} \sum_{\bar{p}_{j+1} \geq 0, p_{j+1} \geq 0, q_{j t} \geq 1}^{\bar{p}_{j+1}+p_{j+1}+\left|q_{j}\right| \leq \bar{N}_{j}} \frac{\bar{N}_{j} !}{\bar{p}_{j+1} ! p_{j+1} ! q_{j} !\left(\bar{N}_{j}-\bar{p}_{j+1}-p_{j+1}-\left|q_{j}\right|\right) !} \\
& \times S_{\bar{N}_{j}}\left[D^{N_{j+1}+\bar{N}_{j}-\bar{p}_{j+1}-p_{j+1}-\left|q_{j}\right|} \mid \cdots\right]\left(D^{\bar{p}_{j+1}} \hat{\nabla}^{\bar{n}_{j+1}} D^{M_{j+1}} a_{r_{j+1}}\right)\left(\hat{\nabla}^{n_{j+10}} \hat{\nabla}^{m_{j+10}} \tau^{E}\right) \\
& \left.\otimes\left(D^{p_{j+1}}\left(\bigotimes_{\kappa=1}^{k_{j+1}} \hat{\nabla}^{1+n_{j+1 \kappa}} \hat{\nabla}^{1+m_{j+1 \kappa} \phi}\right)\right)\left(\bigotimes_{t=1}^{T_{j}} b_{0} D^{q_{j t}} a_{0}\right) b_{0}\right]
\end{aligned}
$$


where $q_{j}=\left(q_{j 1}, \ldots, q_{j T_{j}}\right) \in \mathbf{Z}_{+}^{T_{j}}$. (Here we have used Corollary 4.1 and Lemma 4.1.) We therefore see that the $\left\{\bar{N}_{j}: 1 \leq j \leq J\right\}$ are given recursively by $\bar{N}_{1}=N_{1}$ and

$$
\bar{N}_{j+1}=N_{j+1}+\bar{N}_{j}-\bar{p}_{j+1}-p_{j+1}-\left|q_{j}\right| \text { for all } 1 \leq j \leq J-1
$$

the unique solution of which is

$$
\bar{N}_{j}=N_{j}+\sum_{i=1}^{j-1}\left(N_{i}-\bar{p}_{i+1}-p_{i+1}-\left|q_{i}\right|\right) \text { for all } 1 \leq j \leq J
$$

in agreement with Definition 4.4.

By repeated application of (4.13) for each $1 \leq j \leq J-1$ and use of Lemma 4.2 for $D^{\bar{N}_{J}} b_{0}$, we obtain

$$
\begin{aligned}
& D^{N_{1}}\left[\cdots \left[D ^ { N _ { j } } \left[\cdots \left[\left(D^{N_{J}} b_{0}\right)\left(\hat{\nabla}^{\bar{n}_{J}} D^{M_{J}} a_{r_{J}}\right)\right.\right.\right.\right. \\
& \left.\left.\otimes\left(\hat{\nabla}^{n_{J 0}} \hat{\nabla}^{m_{J 0}} \tau^{E}\right)\left(\bigotimes_{\kappa=1}^{k_{J}} \hat{\nabla}^{1+n_{J \kappa}} \hat{\nabla}^{1+m_{J \kappa}} \phi\right) b_{0}\right] \cdots\right] \\
& \left.\left.\otimes\left(\hat{\nabla}^{\bar{n}_{j}} D^{M_{j}} a_{r_{j}}\right)\left(\hat{\nabla}^{n_{j 0}} \hat{\nabla}^{m_{j 0}} \tau^{E}\right)\left(\bigotimes_{\kappa=1}^{k_{j}} \hat{\nabla}^{1+n_{j \kappa}} \hat{\nabla}^{1+m_{j \kappa} \phi}\right) b_{0}\right] \cdots\right] \\
& \otimes\left(\hat{\nabla}^{\bar{n}_{1}} D^{M_{1}} a_{r_{1}}\right)\left(\hat{\nabla}^{n_{10}} \hat{\nabla}^{m_{10}} \tau^{E}\right)\left(\bigotimes_{\kappa=1}^{k_{1}} \hat{\nabla}^{1+n_{1 \kappa}} \hat{\nabla}^{1+m_{1 \kappa} \phi}\right) b_{0} \\
& =\sum_{T_{1}=0}^{\bar{N}_{1}} \sum_{\bar{p}_{2} \geq 0, p_{2} \geq 0, q_{1 t} \geq 1}^{\bar{p}_{2}+p_{2}+\left|q_{1}\right| \leq \bar{N}_{1}} \sum_{T_{2}=0}^{\bar{N}_{2}} \sum_{\bar{p}_{3} \geq 0, p_{3} \geq 0, q_{2 t} \geq 1}^{\bar{p}_{3}+p_{3}+\left|q_{2}\right| \leq \bar{N}_{2}} \\
& \cdots \sum_{T_{J-1}=0}^{\bar{N}_{J-1}} \sum_{\bar{p}_{J} \geq 0, p_{J} \geq 0, q_{J-1} \geq 1}^{\bar{p}_{J}+p_{J}+\left|q_{J-1}\right| \leq \bar{N}_{J-1}} \sum_{T_{J}=0}^{\bar{N}_{J}} \sum_{q_{J t} \geq 1}^{\left|q_{J}\right|=\bar{N}_{J}} \\
& (-1)^{T_{1}+\cdots+T_{J}} \frac{\bar{N}_{1} !}{\bar{p}_{2} ! p_{2} ! q_{1} !\left(\bar{N}_{2}-N_{2}\right) !} \frac{\bar{N}_{2} !}{\bar{p}_{3} ! p_{3} ! q_{2} !\left(\bar{N}_{3}-N_{3}\right) !} \\
& \cdots \frac{\bar{N}_{J-1} !}{\bar{p}_{J} ! p_{J} ! q_{J-1} !\left(\bar{N}_{J}-N_{J}\right) !} \frac{\bar{N}_{J} !}{q_{J} !}
\end{aligned}
$$




$$
\begin{aligned}
& \times S_{\bar{N}_{1}} \cdots S_{\bar{N}_{J}}\left[\left(\bigotimes_{t=1}^{T_{J}} b_{0} D^{q_{J t}} a_{0}\right) b_{0}\left(D^{\bar{p}_{J}} \hat{\nabla}^{\bar{n}_{J}} D^{M_{J}} a_{r_{J}}\right)\left(\hat{\nabla}^{n_{J 0}} \hat{\nabla}^{m_{J 0}} \tau^{E}\right)\right. \\
& \otimes\left(D^{p_{J}}\left(\bigotimes_{\kappa=1}^{k_{J}} \hat{\nabla}^{1+n_{J \kappa}} \hat{\nabla}^{1+m_{J \kappa}} \phi\right)\right) \cdots\left(\bigotimes_{t=1}^{T_{2}} b_{0} D^{q_{2 t}} a_{0}\right) b_{0}\left(D^{\bar{p}_{2}} \hat{\nabla}^{\bar{n}_{2}} D^{M_{2}} a_{r_{2}}\right) \\
& \otimes\left(\hat{\nabla}^{n_{20}} \hat{\nabla}^{m_{20}} \tau^{E}\right)\left(D^{p_{2}}\left(\bigotimes_{\kappa=1}^{k_{2}} \hat{\nabla}^{1+n_{2 \kappa}} \hat{\nabla}^{1+m_{2 \kappa}} \phi\right)\right)\left(\bigotimes_{t=1}^{T_{1}} b_{0} D^{q_{1 t}} a_{0}\right) \\
& \left.\otimes b_{0}\left(\hat{\nabla}^{\bar{n}_{1}} D^{M_{1}} a_{r_{1}}\right)\left(\hat{\nabla}^{n_{10}} \hat{\nabla}^{m_{10}} \tau^{E}\right)\left(\bigotimes_{\kappa=1}^{k_{1}} \hat{\nabla}^{1+n_{1 \kappa}} \hat{\nabla}^{1+m_{1 \kappa} \phi}\right) b_{0}\right] \\
& |\bar{p}+p|+|q|=|N| \\
& \sum_{i=1}^{j}\left(\bar{p}_{i+1}+p_{i+1}+\left|q_{i}\right|\right) \leq \sum_{i=1}^{j} N_{i} \\
& =\sum_{T_{j} \geq 0} \sum_{\bar{p}_{j} \geq 0, p_{j} \geq 0, q_{t j} \geq 1}^{\sum_{\bar{p}_{1}=p_{1}=0}}(-1)^{|T|} \frac{\bar{N} !}{\bar{p} ! p ! q !(\bar{N}-N) !} S_{\bar{N}_{1}} \cdots S_{\bar{N}_{J}} \\
& {\left[\bigotimes _ { j = 1 } ^ { J } R \left[\left(\bigotimes_{t=1}^{T_{j}} b_{0} D^{q_{j t}} a_{0}\right) b_{0}\left(D^{\bar{p}_{j}} \hat{\nabla}^{\bar{n}_{j}} D^{M_{j}} a_{r_{j}}\right)\left(\hat{\nabla}^{n_{j 0}} \hat{\nabla}^{m_{j 0}} \tau^{E}\right)\right.\right.} \\
& \left.\otimes\left(D^{p_{j}}\left(\bigotimes_{\kappa=1}^{k_{j}} \hat{\nabla}^{1+n_{j \kappa}} \hat{\nabla}^{1+m_{j \kappa} \phi}\right)\right)\right] b_{0}
\end{aligned}
$$

where we have again invoked Remark 4.4 to bring the $T_{j}$ summations to the outside, and have used (4.14) to rewrite the constraints

$$
\bar{p}_{j+1}+p_{j+1}+\left|q_{j}\right| \leq \bar{N}_{j} \text { for all } 1 \leq j \leq J-1
$$

and $\left|q_{J}\right|=\bar{N}_{J}$ as

$$
\sum_{i=1}^{j}\left(\bar{p}_{i+1}+p_{i+1}+\left|q_{i}\right|\right) \leq \sum_{i=1}^{j} N_{i} \text { for all } 1 \leq j \leq J-1
$$

and $|\bar{p}+p|+|q|=|N|$, respectively.

Substituting (4.15) in (4.9) gives, if $s \geq 1$,

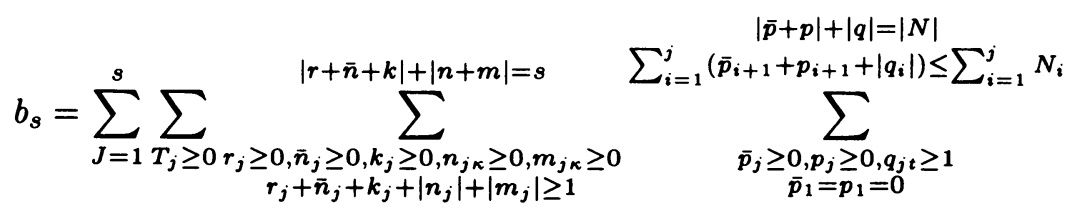

$$
\begin{aligned}
& i^{2|T|+2 J+|r|-s} \bar{N} !\left(\bar{n} ! k ! n_{+} ! m_{+} ! \bar{p} ! p ! q !(\bar{N}-N) !\right)^{-1} \\
& \times\left[\bigotimes _ { j = 1 } ^ { J } R \left[\left(\bigotimes_{t=1}^{T_{j}} b_{0} D^{q_{t j}} a_{0}\right) b_{0}\left(D^{\bar{p}_{j}} \hat{\nabla}^{\bar{n}_{j}} D^{M_{j}} a_{r_{j}}\right)\left(\hat{\nabla}^{n_{j 0}} \hat{\nabla}^{m_{j 0}} \tau^{E}\right)\right.\right. \\
&\left.\otimes\left(D^{p_{j}}\left(\bigotimes_{\kappa=1}^{k_{j}} \hat{\nabla}^{1+n_{j \kappa}} \hat{\nabla}^{1+m_{j \kappa} \phi}\right)\right)\right] b_{0}
\end{aligned}
$$


in which the various factors are contracted as specified in Theorem 4.1.

By combining the lower with the upper bounds in the last summation above we find that

$$
\sum_{i=1}^{j} T_{i} \leq \sum_{i=1}^{j}\left(\bar{p}_{i+1}+p_{i+1}+\left|q_{i}\right|\right) \leq \sum_{i=1}^{j} N_{i} \quad \text { for all } 1 \leq j \leq J-1
$$

and

$$
|T| \leq|\bar{p}+p|+|q|=|N| .
$$

Hence the last summation is vacuous unless

$$
\sum_{i=1}^{j} N_{i} \geq \sum_{i=1}^{j} T_{i} \text { for all } 1 \leq j \leq J,
$$

which becomes a new lower bound on the preceding summation.

Furthermore, from the constraints

$$
r_{j}+\bar{n}_{j}+k_{j}+\left|n_{j}\right|+\left|m_{j}\right| \geq 1 \text { for all } 1 \leq j \leq J
$$

and

$$
|r+\bar{n}+k|+|n+m|=s
$$

we learn, for all $1 \leq j \leq J$,

$$
\begin{aligned}
\sum_{i=1}^{j} N_{i} & \leq \sum_{i=1}^{j}\left(r_{i}+\bar{n}_{i}+k_{i}+\left|n_{i}\right|+\left|m_{i}\right|\right) \\
& =s-\sum_{i=j+1}^{J}\left(r_{i}+\bar{n}_{i}+k_{i}+\left|n_{i}\right|+\left|m_{i}\right|\right) \leq s-(J-j) .
\end{aligned}
$$

Thus the summation over the variables $r_{j}, \bar{n}_{j}, k_{j}, n_{j \kappa}$, and $m_{j \kappa}$ is vacuous unless

$$
\sum_{i=1}^{j} T_{i} \leq s-J+j \quad \text { for all } 1 \leq j \leq J,
$$

which becomes an upper bound on the $T_{j}$ summation.

(4.12) now follows from (4.16), (4.17), and (4.18).

Upon specialization to a differential operator Theorem 4.1 yields

THEOREM 4.2. Let $A=\sum_{r=0}^{l} A_{r} \hat{\nabla}^{l-r}$, where

$$
A_{r} \in \Gamma^{\infty}\left(\operatorname{End}(E) \otimes S\left(\bigotimes^{l-r}(T(M))\right)\right),
$$

be a differential operator of order $l>0$ which is elliptic with respect to a ray $\Gamma$ in $\mathbf{C}$, let $\lambda \in \Gamma$, and let $B_{\lambda} \in L^{-l}(M, E, E)$ be a resolvent parametrix of $A$. The intrinsic symbol of $B_{\lambda}$ has asymptotic expansion $b \sim \sum_{s \geq 0} b_{s}$, where $b_{0}=\left(A\left(\bigotimes^{l}(i \xi)\right)-\lambda\right)^{-1}$ 
and, for all $s \geq 1$,

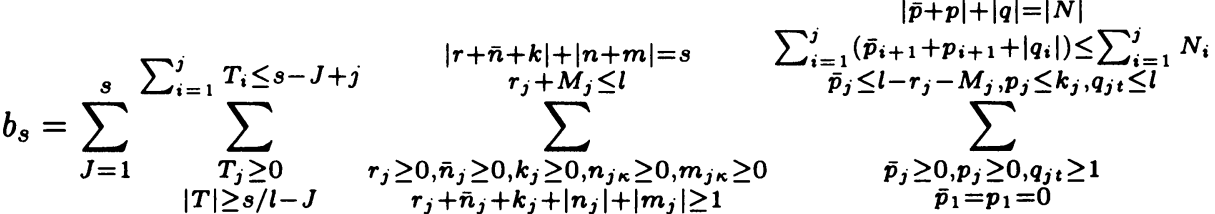

$$
\begin{aligned}
& \sum_{i=1}^{j} N_{i} \geq \sum_{i=1}^{j} T_{i} \\
& i^{(l+2)(J+|T|)-s} \frac{\bar{N} !}{(\bar{N}-N) ! \bar{n} ! n_{+} !} \frac{(l-r) !}{(l-r-M-\bar{p}) ! m_{+} ! \bar{p} !} \frac{(l !)^{|T|}}{(l-q) ! q !} \frac{1}{(k-p) ! p !} \\
& \times\left[\bigotimes _ { j = 1 } ^ { J } R \left[\left(\bigotimes_{t=1}^{T_{j}} b_{0} A_{0}\right) b_{0}\left(\hat{\nabla}^{\bar{n}_{j}} A_{r_{j}}\right)\left(\hat{\nabla}^{n_{j 0}} \hat{\nabla}^{m_{j 0}} \tau^{E}\right)\right.\right. \\
& \left.\left.\otimes\left(S_{k_{j}}\left(\bigotimes_{\kappa=1}^{k_{j}} \hat{\nabla}^{1+n_{j \kappa}} \hat{\nabla}^{1+m_{j \kappa}} \Phi\right)\right)\right]\right] b_{0}\left(\bigotimes^{l(J+|T|)-s} \xi\right) .
\end{aligned}
$$

In (4.19), all subscripted quantities are as listed in Definitions 4.3 and 4.4, $l-r=\left(l-r_{1}, \ldots, l-r_{J}\right) \in \mathbf{Z}_{+}^{J}, l-q=\left(l-q_{j t}\right)$, where $1 \leq j \leq J$ and $1 \leq t \leq T_{j}, A_{r_{j}}$ has an implicit right composition with $p$ understood, $S_{k_{j}}$ indicates symmetrization in the $k_{j}$ contravariant indices of $\bigotimes_{\kappa=1}^{k_{j}} \hat{\nabla}^{1+n_{j \kappa}} \hat{\nabla}^{1+m_{j \kappa}} \Phi$, and $\bigotimes^{l(J+|T|)-s} \xi$ abbreviates the mapping $\xi \mapsto \bigotimes^{l(J+|T|)-s} \xi$. The (inner) covariant derivatives of the form $\hat{\nabla}^{m_{j 0}}$ and $\hat{\nabla}^{1+m_{j \kappa}}$ are contracted with $M_{j}$ of the $l-r_{j}$ contravariant indices of $\hat{\nabla}^{\bar{n}_{j}} A_{r_{j}}$ for each $1 \leq j \leq J$. The (outer) covariant derivatives of the form $\hat{\nabla}^{\bar{n}_{j}}, \hat{\nabla}^{n_{j 0}}$, and $\hat{\nabla}^{1+n_{j \kappa}}$ have much more complicated contractions, which are described inductively as follows: For each $1 \leq j \leq J-1$, the $N_{j}$ covariant derivatives of the form $\hat{\nabla}^{\bar{n}_{j}}, \hat{\nabla}^{n_{j 0}}$, and $\hat{\nabla}^{1+n_{j \kappa}}$ are combined with the $\sum_{i=1}^{j-1}\left(N_{i}-\bar{p}_{i+1}-p_{i+1}-\left|q_{i}\right|\right)$ as yet uncontracted covariant derivatives of the form $\hat{\nabla}^{\bar{n}_{i}}, \hat{\nabla}^{n_{i 0}}$, and $\hat{\nabla}^{1+n_{i \kappa}}$ for all $1 \leq i \leq j-1$, and the total of $\bar{N}_{j}=N_{j}+\sum_{i=1}^{j-1}\left(N_{i}-\bar{p}_{i+1}-p_{i+1}-\left|q_{i}\right|\right)$ covariant derivatives is symmetrized. Of these, $q_{j t}$ are contracted with $q_{j t}$ of the $l$ contravariant indices of the th $A_{0}$ factor in $\bigotimes_{t=1}^{T_{j}} b_{0} A_{0}$ for each $1 \leq t \leq T_{j}, p_{j+1}$ are contracted with $p_{j+1}$ of the $k_{j+1}$ contravariant indices of $S_{k_{j+1}}\left(\bigotimes_{\kappa=1}^{k_{j+1}} \hat{\nabla}^{1+n_{j+1 \kappa}} \hat{\nabla}^{1+m_{j+1 \kappa}} \Phi\right)$, and $\bar{p}_{j+1}$ are contracted with $\bar{p}_{j+1}$ of the $l-r_{j+1}-M_{j+1}$ as yet uncontracted contravariant indices of $\hat{\nabla}^{\bar{n}_{j+1}} A_{r_{j+1}}$. The remaining

$$
\bar{N}_{j}-\bar{p}_{j+1}-p_{j+1}-\left|q_{j}\right|=\sum_{i=1}^{j}\left(N_{i}-\bar{p}_{i+1}-p_{i+1}-\left|q_{i}\right|\right)
$$

covariant derivatives are combined with the $N_{j+1}$ covariant derivatives of the form $\hat{\nabla}^{\bar{n}_{j+1}}, \hat{\nabla}^{n_{j+10}}$, and $\hat{\nabla}^{1+n_{j+1 \kappa}}$, and the process is repeated. After the final such operation (when $j=J-1$ ), the remaining

$$
\bar{N}_{J-1}-\bar{p}_{J}-p_{J}-\left|q_{J-1}\right|=\sum_{i=1}^{J-1}\left(N_{i}-\bar{p}_{i+1}-p_{i+1}-\left|q_{i}\right|\right)
$$


covariant derivatives are combined with the $N_{J}$ covariant derivatives of the form $\hat{\nabla}^{\bar{n}_{J}}, \hat{\nabla}^{n_{J 0}}$, and $\hat{\nabla}^{1+n_{J \kappa}}$, and the total of

$$
\bar{N}_{J}=N_{J}+\sum_{i=1}^{J-1}\left(N_{i}-\bar{p}_{i+1}-p_{i+1}-\left|q_{i}\right|\right)=\left|q_{J}\right|
$$

covariant derivatives is symmetrized. Of these, $q_{J t}$ are contracted with $q_{J t}$ of the $l$ contravariant indices of the th $A_{0}$ factor in $\otimes_{t=1}^{T_{J}} b_{0} A_{0}$ for each $1 \leq t \leq T_{J}$. Finally, $\bigotimes^{l(J+|T|)-s} \xi$ is contracted with the remaining $l(J+|T|)-s$ uncontracted contravariant indices.

PrOOF. By Proposition 3.3,

$$
D^{q_{j t}} a_{0}= \begin{cases}\frac{i^{l} l !}{\left(l-q^{\prime \prime} j t\right) !} A_{0}\left(\bigotimes^{l-q_{j t}} \xi\right) & \text { if } q_{j t} \leq l \\ 0 & \text { if } q_{j t}>l\end{cases}
$$

and

$$
\begin{aligned}
& D^{\bar{p}_{j}} \hat{\nabla}^{\bar{n}_{j}} D^{M_{j}} a_{r_{j}}=D^{M_{j}+\bar{p}_{j}} \hat{\nabla}^{\bar{n}_{j}} a_{r_{j}}=D^{M_{j}+\bar{p}_{j}}\left[\left(\hat{\nabla}^{\bar{n}_{j}} A_{r_{j}}\right)\left(\bigotimes^{l-r_{j}}(i \xi)\right)\right] \\
& \quad= \begin{cases}\frac{i^{l-r_{j}}\left(l-r_{j}\right) !}{\left(l-r_{j}-M_{j}-\bar{p}_{j}\right) !}\left(\hat{\nabla}^{\bar{n}_{j}} A_{r_{j}}\right)\left(\bigotimes^{l-r_{j}-M_{j}-\bar{p}_{j}} \xi\right) & \text { if } M_{j}+\bar{p}_{j} \leq l-r_{j}, \\
0 & \text { if } M_{j}+\bar{p}_{j}>l-r_{j} .\end{cases}
\end{aligned}
$$

Also,

$$
\begin{aligned}
& D^{p_{j}}\left(\bigotimes_{\kappa=1}^{k_{j}} \hat{\nabla}^{1+n_{j \kappa}} \hat{\nabla}^{1+m_{j \kappa} \phi}\right) \\
& =D^{p_{j}}\left[\left(S_{k_{j}}\left(\bigotimes_{\kappa=1}^{k_{j}} \hat{\nabla}^{1+n_{j \kappa}} \hat{\nabla}^{1+m_{j \kappa} \Phi}\right)\right)\left(\bigotimes^{k_{j}} \xi\right)\right] \\
& = \begin{cases}\frac{k_{j} !}{\left(k_{j}-p_{j}\right) !}\left(S_{k_{j}}\left(\bigotimes_{\kappa=1}^{k_{j}} \hat{\nabla}^{1+n_{j \kappa}} \hat{\nabla}^{1+m_{j \kappa}} \Phi\right)\right)\left(\bigotimes^{k_{j}-p_{j}} \xi\right) & \text { if } p_{j} \leq k_{j}, \\
0 & \text { if } p_{j}>k_{j} .\end{cases}
\end{aligned}
$$

Substituting these expressions in the summand of (4.12) gives

$$
\begin{aligned}
& i^{2(J+|T|)+|r|-s} \bar{N} !\left(\bar{n} ! k ! n_{+} ! m_{+} ! \bar{p} ! p ! q !(\bar{N}-N) !\right)^{-1} \\
& \times \prod_{j=1}^{J}\left[\left(\prod_{t=1}^{T_{j}} \frac{i^{l} l !}{\left(l-q_{j t}\right) !}\right) \frac{i^{l-r_{j}}\left(l-r_{j}\right) !}{\left(l-r_{j}-M_{j}-\bar{p}_{j}\right) !} \frac{k_{j} !}{\left(k_{j}-p_{j}\right) !}\right] \\
& \times\left[\bigotimes _ { j = 1 } ^ { J } R \left[\left(\bigotimes_{t=1}^{T_{j}} b_{0} A_{0}\left(\bigotimes^{l-q_{j t}} \xi\right)\right) b_{0}\left(\hat{\nabla}^{\bar{n}_{j}} A_{r_{j}}\right)\left(\bigotimes^{l-r_{j}-M_{j}-\bar{p}_{j}} \xi\right)\left(\hat{\nabla}^{n_{j 0}} \hat{\nabla}^{m_{j 0}} \tau^{E}\right)\right.\right. \\
& \left.\left.\otimes\left(S_{k_{j}}\left(\bigotimes_{\kappa=1}^{k_{j}} \hat{\nabla}^{1+n_{j \kappa}} \hat{\nabla}^{1+m_{j \kappa} \Phi}\right)\right)\left(\bigotimes^{k_{j}-p_{j}} \xi\right)\right]\right] b_{0}
\end{aligned}
$$


Thus (4.12) becomes

$$
\begin{aligned}
& b_{s}=\sum_{J=1}^{s} \sum_{T_{j} \geq 0}^{\sum_{i=1}^{j} T_{i} \leq s-J+j} \sum_{r_{j} \geq 0, \bar{n}_{j} \geq 0, k_{j} \geq 0, n_{j \kappa} \geq 0, m_{j \kappa} \geq 0}^{|r+\bar{n}+k|+|n+m|=s} \\
& r_{j}+\bar{n}_{j}+k_{j}+\left|n_{j}\right|+\left|m_{j}\right| \geq 1 \\
& \sum_{i=1}^{j} N_{i} \geq \sum_{i=1}^{j} T_{i} \\
& |\bar{p}+p|+|q|=|N| \\
& \sum_{i=1}^{j}\left(\bar{p}_{i+1}+p_{i+1}+\left|q_{i}\right|\right) \leq \sum_{i=1}^{j} N_{i} \\
& \times \sum_{\substack{\bar{p}_{j} \geq 0, p_{j} \geq 0, q_{j} \geq 1 \\
\bar{p}_{1}=p_{1}=0}} i^{(l+2)(J+|T|)-s}(l !)^{|T|} \bar{N} !(l-r) ! \\
& \times\left(\bar{n} ! n_{+} ! m_{+} ! \bar{p} ! p ! q !(\bar{N}-N) !(l-q) !(l-r-M-\bar{p}) !(k-p) !\right)^{-1} \\
& \times\left[\bigotimes _ { j = 1 } ^ { J } R \left[\left(\bigotimes_{t=1}^{T_{j}} b_{0} A_{0}\right) b_{0}\left(\hat{\nabla}^{\bar{n}_{j}} A_{r_{j}}\right)\left(\hat{\nabla}^{n_{j 0}} \hat{\nabla}^{m_{j 0}} \tau^{E}\right)\right.\right. \\
& \left.\otimes\left(S_{k_{j}}\left(\bigotimes_{\kappa=1}^{k_{j}} \hat{\nabla}^{1+n_{j \kappa} \hat{\nabla}^{1+m_{j \kappa} \Phi}}\right)\right)\right] b_{0}\left(\bigotimes^{l(J+|T|)-s} \xi\right),
\end{aligned}
$$

where all explicit $\xi$ factors, of which the total number is

$$
\begin{aligned}
& \sum_{j=1}^{J}\left(\sum_{t=1}^{T_{j}}\left(l-q_{j t}\right)+l-r_{j}-M_{j}-\bar{p}_{j}+k_{j}-p_{j}\right) \\
& =\sum_{j=1}^{J}\left(l\left(1+T_{j}\right)-\left(r_{j}+\left|m_{j}\right|+\bar{p}_{j}+p_{j}+\left|q_{j}\right|\right)\right) \\
& \quad=l(J+|T|)-(|r|+|m|+|N|)=l(J+|T|)-s,
\end{aligned}
$$

have been isolated on the right, and the various indices are contracted as specified in Theorem 4.2 .

The bounds on the last summation in (4.20) imply that it will be vacuous unless

$$
0 \leq \bar{p}_{j} \leq l-r_{j}-M_{j} \text { for all } 1 \leq j \leq J
$$

and

$$
|N|=|\bar{p}+p|+|q| \leq l J-|r|-|M|+|k|+l|T| .
$$

(4.21) implies

$$
r_{j}+M_{j} \leq l \text { for all } 1 \leq j \leq J,
$$

which becomes a new upper bound on the preceding summation. (4.22) implies

$$
|r+\bar{n}+k|+|n+m| \leq l(J+|T|)
$$

or, since $|r+\bar{n}+k|+|n+m|=s$,

$$
|T| \geq s / l-J,
$$

which becomes a new lower bound on the $T_{j}$ summation in (4.20). 
(4.19) now follows from (4.20), (4.23), and (4.24).

REMARK 4.5. Since $|T| \in \mathbf{Z}$, (4.24) may be rewritten as $|T| \geq\{s / l\}-J$, where $\{x\}=-[-x]$ denotes the smallest integer not less than $x \in \mathbf{R} ;$ this alternative form for the lower bound could be used in the $T_{j}$ summation in (4.19).

REMARK 4.6. That the number of explicit $\xi$ factors isolated on the right of (4.19) should equal $l(J+|T|)-s$ can be understood from the fact that $b_{s}$ is positivehomogeneous of degree $-l-s$ in $\left(\xi, \lambda^{1 / l}\right)$ for all $\xi \in T^{*}(M) \backslash 0, \lambda \in \Gamma$, and $s \in \mathbf{Z}_{+}$, as established in Proposition 4.1. The summand of (4.19) contains $J+|T|+1$ factors of $b_{0}$, which alone imply a positive-homogeneity degree of $-l(J+|T|+1)$; the $\otimes^{l(J+|T|)-s} \xi$ factor is required in order to give the correct overall degree of positive-homogeneity. Notice, in addition, that $\bigotimes^{l(J+|T|)-s} \xi$ only has sense if $l(J+|T|) \geq s$, which is just the reason behind the constraint (4.24).

REMARK 4.7. Let $U=J+|T|$. The previous remark shows that the $U$ of a term determines its structure as a function of $\xi$. Moreover, $U$ is the total number of coefficient tensors, $\hat{\nabla}^{r} A_{s}$, in the term (where $r \geq 0$ and $s \geq 0$ ). Arguably, therefore, $U$ describes the most important qualitative features of a term, and it may be desirable to recast the summation in (4.19) in this way:

$$
\sum_{J=1}^{s} \sum_{\substack{T_{j} \geq 0 \\|T| \geq s / l-J}}^{\sum_{i=1}^{j}}[\cdots]=\sum_{U=\{s / l\}}^{2 s} \sum_{J=\max (1, U-s)}^{\min (U, s)} \sum_{T_{j} \geq 0}^{\sum_{i=1}^{j} \sum_{i}-J+j}[\cdots] .
$$

REMARK 4.8. In (4.19) the factorials have been grouped, as far as possible, into combinations which are integers. These are essentially multinomial coefficients, that is, quantities of the general form

$$
\left(\sum_{j=1}^{p} n_{j}\right) ! / \prod_{j=1}^{p} n_{j} !
$$

which are the cardinalities of factor spaces of permutation groups.

5. Ordering the summations. In the main results of the previous section, Theorems 4.1 and 4.2 , the summations over the variables $r_{j}, \bar{n}_{j}, k_{j}, n_{j \kappa}$, and $m_{j \kappa}$ were handled by one large multi-index summation. This approach offers two advantages: first, it makes the already cumbersome formulas easier to write; second, with the constraint $|r+\bar{n}+k|+|n+m|=s$ it allows the summations over these variables to be interpreted as a summation over distinct arrangements of $s$ elements among the entries $r_{j}, \bar{n}_{j}, k_{j}, n_{j \kappa}$, and $m_{j \kappa}$ in some large multi-index array. However, it will prove useful in later developments to introduce a canonical ordering of the summations over these variables and to make the individual summations as "tight" as possible (to avoid running through values for one variable which cause a summation over another to be vacuous): both topics are addressed in this section.

In particular, we envisage the generation of the terms in $b_{s}$ (or in some related object, such as a term of the heat-kernel expansion) by a computer-see $\S 7$. It is clear that a canonical ordering is necessary to specify an algorithm for such a task; for aesthetic and practical reasons one will choose an ordering in which the most important structural features of a term are determined by the multi-indices 
standing furthest to the outside in the multiple summation, so that like terms will be grouped together (cf. Remark 4.7). It is also clear that tight summations are highly desirable for producing an efficient program.

The following lemmas will be useful in obtaining the main result of this section, Proposition 5.1.

LEMMA 5.1. In the summations over the variables $r_{j}, \bar{n}_{j}, k_{j}, n_{j \kappa}$, and $m_{j \kappa}$ in Theorems 4.1 and 4.2, we may replace the constraint

$$
r_{j}+\bar{n}_{j}+k_{j}+\left|n_{j}\right|+\left|m_{j}\right| \geq 1 \text { for all } 1 \leq j \leq J
$$

by

$$
r_{j}+\bar{n}_{j}+k_{j}+n_{j 0}+m_{j 0} \geq 1 \text { for all } 1 \leq j \leq J
$$

and may impose the additional constraints

$$
m_{j 0} \geq 1-\delta_{n_{j 0}, 0} \quad \text { for all } 1 \leq j \leq J
$$

and

$$
n_{j 0} \geq 1-\delta_{m_{j 0}, 0} \text { for all } 1 \leq j \leq J
$$

(that is, $m_{j 0}$ and $n_{j 0}$ are either both zero or both nonzero).

ProOF. Since $\left|n_{j}\right| \geq n_{j 0}$ and $\left|m_{j}\right| \geq m_{j 0}$, (5.2) implies (5.1). Also, the negation of (5.2) holds if and only if $r_{j}=\bar{n}_{j}=k_{j}=n_{j 0}=m_{j 0}=0$, which implies $r_{j}=\bar{n}_{j}=k_{j}=\left|n_{j}\right|=\left|m_{j}\right|=0$, and this is so if and only if the negation of (5.1) holds. Hence (5.1) and (5.2) are equivalent.

By Proposition 2.2, the summands in Theorems 4.1 and 4.2, which involve $\hat{\nabla}^{n_{j 0}} \hat{\nabla}^{m_{j 0}} \tau^{E}$ factors for all $1 \leq j \leq J$, will vanish unless either $n_{j 0}=0=m_{j 0}$ or both $n_{j 0} \geq 1$ and $m_{j 0} \geq 1$ for each $1 \leq j \leq J$. The constraints (5.3) and (5.4) restrict us to such values of $n_{j 0}$ and $m_{j 0}$ : for example, if $n_{j 0} \geq 1$ then (5.3) requires $m_{j 0} \geq 1$ while (5.4) is satisfied for any $m_{j 0}$; on the other hand, if $n_{j 0}=0$ then (5.4) requires $m_{j 0}=0$ while (5.3) is satisfied for any $m_{j 0}$.

LEMMA 5.2. The constraints (5.3) and (5.4) imply that (5.2) may be replaced by

$$
n_{j 0} \geq \delta_{r_{j}+\bar{n}_{j}+k_{j}, 0} \quad \text { for all } 1 \leq j \leq J .
$$

PROOF. If $r_{j}+\bar{n}_{j}+k_{j} \geq 1$ then (5.2) is satisfied for any $n_{j 0}$ and $m_{j 0}$. If $r_{j}+\bar{n}_{j}+k_{j}=0$ then it reduces to $n_{j 0}+m_{j 0} \geq 1$, which is compatible with (5.3) and (5.4) only if both $n_{j 0} \geq 1$ and $m_{j 0} \geq 1$. The constraint on $n_{j 0}$ is furnished by (5.5); that on $m_{j 0}$ then follows from (5.3) and (5.4). 
PROPOSITION 5.1. The summations over the variables $r_{j}, \bar{n}_{j}, k_{j}, n_{j \kappa}$, and $m_{j \kappa}$ in Theorems 4.1 and 4.2 may be ordered as

$$
\begin{aligned}
& \sum_{r_{j} \geq 0, \bar{n}_{j} \geq 0, k_{j} \geq 0, n_{j \kappa} \geq 0, m_{j \kappa} \geq 0}^{|r+\bar{n}+k|+|n+m|=s}[\cdots]=\sum_{r_{j} \geq 0}^{|r|+Z_{j}(T, r) \leq s} \sum_{\bar{n}_{j} \geq 0}^{|\bar{n}|+Z_{j}(T-\bar{n}, r+\bar{n}) \leq s-|r|} \\
& r_{j}+\bar{n}_{j}+k_{j}+\left|n_{j}\right|+\left|m_{j}\right| \geq 1 \\
& \sum_{i=1}^{j} N_{i} \geq \sum_{i=1}^{j} T_{i} \\
& |k|+Z_{j}(T-\bar{n}-k, r+\bar{n}+k)+Z(r+\bar{n}+k) \leq s-|r+\bar{n}| \\
& \sum_{k_{j} \geq 0} \\
& \times \sum_{\substack{n_{j \kappa} \geq 0, n_{j 0} \geq \delta_{r_{j}+\tilde{n}_{j}+k_{j}, 0} \\
\sum_{i=1}^{j}\left|n_{i}\right| \geq \sum_{i=1}^{j}\left(T_{i}-\bar{n}_{i}-k_{i}\right)}}^{|n|+J-Z\left(n_{0}\right) \leq s-|r+\bar{n}+k|} \sum_{m_{j \kappa} \geq 0, m_{j 0} \geq 1-\delta_{n_{j 0}, 0}}^{\substack{|m|=s-|r+\bar{n}+k|-|n| \\
1-\delta_{m_{j 0}, 0 \leq n_{j 0}}}}[\cdots]
\end{aligned}
$$

and

$$
\begin{aligned}
& \sum_{r_{j} \geq 0, \bar{n}_{j} \geq 0, k_{j} \geq 0, n_{j \kappa} \geq 0, m_{j \kappa} \geq 0}^{|r+\bar{n}+k|+|n+m|=s, r_{j}+M_{j} \leq l}[\cdots]=\sum_{r_{j} \geq 0}^{|r|+Z_{j}(T, r) \leq s} \sum_{\bar{n}_{j} \geq 0}^{r_{j} \leq l}|\bar{n}|+Z_{j}(T-\bar{n}, r+\bar{n}) \leq s-|r| \\
& r_{j}+\bar{n}_{j}+k_{j}+\left|n_{j}\right|+\left|m_{j}\right| \geq 1 \\
& \sum_{i=1}^{j} N_{i} \geq \sum_{i=1}^{j} T_{i} \\
& |k|+Z_{j}(T-\bar{n}-k, r+\bar{n}+k)+Z(r+\bar{n}+k) \leq s-|r+\bar{n}| \\
& \times \quad \sum_{k_{j} \geq 0}^{k \leq l-r_{j}} \\
& |n|+J-Z\left(n_{0}\right) \leq s-|r+\bar{n}+k| \quad\left|m_{j}\right| \leq l-r_{j}-k_{j} \\
& 1-\delta_{n_{j 0}, 0} \leq l-r_{j}-k_{j} \quad 1-\delta_{m_{j 0}, 0} \leq n_{j 0} \\
& \times \sum_{n_{j \kappa} \geq 0, n_{j 0} \geq \delta_{r_{j}+\bar{n}_{j}+k_{j}, 0}} \sum_{m_{j \kappa} \geq 0, m_{j 0} \geq 1-\delta_{n_{j 0}, 0}}[\cdots] \text {, } \\
& \begin{array}{c}
\sum_{i=1}^{j}\left|n_{i}\right| \geq \sum_{i=1}^{j}\left(T_{i}-\bar{n}_{i}-k_{i}\right) \\
|n| \geq s-l J-|\bar{n}|
\end{array}
\end{aligned}
$$

respectively, where $[\cdots]$ indicates the appropriate summand, $Z_{j}(\alpha, \beta)$ and $Z(\beta)$ are defined for any multi-indices $\alpha=\left(\alpha_{1}, \ldots, \alpha_{J}\right) \in \mathbf{Z}_{+}^{J}$ and $\beta=\left(\beta_{1}, \ldots, \beta_{J}\right) \in \mathbf{Z}_{+}^{J}$ by

$$
Z_{j}(\alpha, \beta)=\sum_{i=1}^{j} \alpha_{i}+\sum_{i=j+1}^{J} \delta_{\beta_{i}, 0} \quad \text { for all } 0 \leq j \leq J
$$

and

$$
Z(\beta)=\sum_{i=1}^{J} \delta_{\beta_{i}, 0}
$$

and summation constraints involving $Z_{j}$ are understood to extend over $0 \leq j \leq J$.

ProOF. First, consider (5.6). Rewriting $\sum_{i=1}^{j} N_{i} \geq \sum_{i=1}^{j} T_{i}$ as

$$
\sum_{i=1}^{j}\left|n_{i}\right| \geq \sum_{i=1}^{j}\left(T_{i}-\bar{n}_{i}-k_{i}\right) \quad \text { for all } 1 \leq j \leq J,
$$


and using Lemmas 5.1 and 5.2 , we obtain

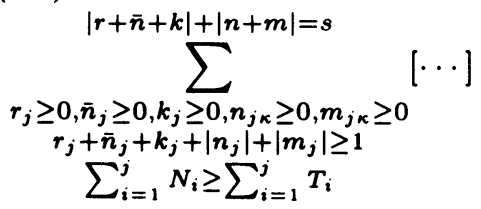

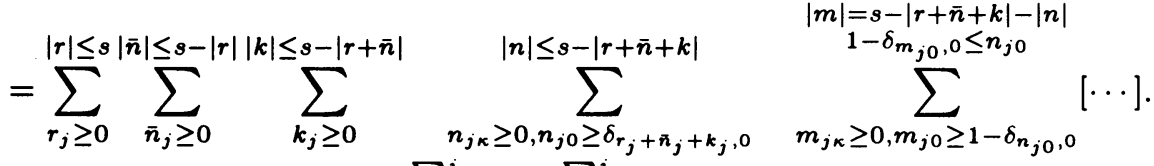

$$
\begin{aligned}
& \sum_{i=1}^{j}\left|n_{i}\right| \geq \sum_{i=1}^{j}\left(T_{i}-\bar{n}_{i}-k_{i}\right)
\end{aligned}
$$

By comparing its upper and lower bounds, we find that the $m_{j \kappa}$ summation is vacuous unless

$$
\sum_{j=1}^{J}\left(1-\delta_{n_{j 0}, 0}\right) \leq|m|=s-|r+\bar{n}+k|-|n|
$$

which implies

$$
|n|+J-Z\left(n_{0}\right) \leq s-|r+\bar{n}+k| .
$$

This becomes a new upper bound for the $n_{j \kappa}$ summation.

From the lower bounds on the $n_{j \kappa}$ summation it follows that

$$
\begin{aligned}
|n| & =\sum_{i=1}^{j}\left|n_{i}\right|+\sum_{i=j+1}^{J}\left|n_{i}\right| \\
& \geq \sum_{i=1}^{j}\left(T_{i}-\bar{n}_{i}-k_{i}\right)+\sum_{i=j+1}^{J} \delta_{r_{i}+\bar{n}_{i}-k_{i}, 0} \quad \text { for all } 0 \leq j \leq J
\end{aligned}
$$

and

$$
1-\delta_{n_{j 0}, 0} \geq \delta_{r_{j}+\bar{n}_{j}+k_{j}, 0} \text { for all } 1 \leq j \leq J .
$$

Thus, the $n_{j \kappa}$ summation is vacuous unless

$$
\begin{aligned}
\sum_{i=1}^{j}\left(T_{i}-\bar{n}_{i}-k_{i}\right)+\sum_{i=j+1}^{J} \delta_{r_{i}+\bar{n}_{i}+k_{i}, 0}+\sum_{i=1}^{J} \delta_{r_{i}+\bar{n}_{i}+k_{i}, 0} & \leq|n|+J-Z\left(n_{0}\right) \\
& \leq s-|r+\bar{n}+k|
\end{aligned}
$$

for all $0 \leq j \leq J$, which implies

(5.13) $|k|+Z_{j}(T-\bar{n}-k, r+\bar{n}+k)+Z(r+\bar{n}+k) \leq s-|r+\bar{n}|$ for all $0 \leq j \leq J$.

This becomes a new family of upper bounds for the $k_{j}$ summation.

Since

$$
|k|+Z_{j}(T-\bar{n}-k, r+\bar{n}+k)=\sum_{i=1}^{j}\left(T_{i}-\bar{n}_{i}\right)+\sum_{i=j+1}^{J}\left(k_{i}+\delta_{r_{i}+\bar{n}_{i}+k_{i}, 0}\right)
$$


and

$$
k_{j}+\delta_{r_{j}+\bar{n}_{j}+k_{j}, 0} \geq \delta_{r_{j}+\bar{n}_{j}, 0} \text { for all } 1 \leq j \leq J
$$

it follows that the $k_{j}$ summation is vacuous unless

$$
\begin{aligned}
\sum_{i=1}^{j}\left(T_{i}-\bar{n}_{i}\right)+\sum_{i=j+1}^{J} \delta_{r_{i}+\bar{n}_{i}, 0} & \leq|k|+Z_{j}(T-\bar{n}-k, r+\bar{n}+k)+Z(r+\bar{n}+k) \\
& \leq s-|r+\bar{n}|
\end{aligned}
$$

for all $0 \leq j \leq J$, which implies

$$
|\bar{n}|+Z_{j}(T-\bar{n}, r+\bar{n}) \leq s-|r| \text { for all } 0 \leq j \leq J .
$$

This becomes a new family of upper bounds for the $\bar{n}_{j}$ summation.

Since

$$
|\bar{n}|+Z_{j}(T-\bar{n}, r+\bar{n})=\sum_{i=1}^{j} T_{i}+\sum_{i=j+1}^{J}\left(\bar{n}_{i}+\delta_{r_{i}+\bar{n}_{i}, 0}\right) \quad \text { for all } 0 \leq j \leq J
$$

and

$$
\bar{n}_{j}+\delta_{r_{j}+\bar{n}_{j}, 0} \geq \delta_{r_{j}, 0} \text { for all } 1 \leq j \leq J,
$$

it follows that the $\bar{n}_{j}$ summation is vacuous unless

$$
\sum_{i=1}^{j} T_{i}+\sum_{i=j+1}^{J} \delta_{r_{i}, 0} \leq|\bar{n}|+Z_{j}(T-\bar{n}, r+\bar{n}) \leq s-|r| \quad \text { for all } 0 \leq j \leq J
$$

which implies

$$
|r|+Z_{j}(T, r) \leq s \quad \text { for all } 0 \leq j \leq J
$$

This becomes a new family of upper bounds for the $r_{j}$ summation.

(5.6) now follows from (5.9), (5.10), (5.13), (5.14), and (5.15).

Next, consider (5.7). Rewriting $r_{j}+M_{j} \leq l$ as

$$
\left|m_{j}\right| \leq l-r_{j}-k_{j} \quad \text { for all } 1 \leq j \leq J,
$$

and attaching this upper bound to the $m_{j \kappa}$ summation in (5.6), we find that the latter is vacuous unless

$$
1-\delta_{n_{j 0}, 0} \leq\left|m_{j}\right| \leq l-r_{j}-k_{j} \text { for all } 1 \leq j \leq J
$$

and

$$
\sum_{j=1}^{J}\left(l-r_{j}-k_{j}\right) \geq|m|=s-|r+\bar{n}+k|-|n|
$$

which imply

$$
1-\delta_{n_{j 0}, 0} \leq l-r_{j}-k_{j} \text { for all } 1 \leq j \leq J
$$

and

$$
|n| \geq s-l J-|\bar{n}|
$$

respectively. These become new bounds for the $n_{j \kappa}$ summation in (5.6). 
From $(5.10),(5.12),(5.17)$, and (5.18), it follows that the $n_{j \kappa}$ summation is vacuous unless

$$
\delta_{r_{j}+\bar{n}_{j}+k_{j}, 0} \leq 1-\delta_{n_{j 0}, 0} \leq l-r_{j}-k_{j} \text { for all } 1 \leq j \leq J
$$

and

$$
s-l J-|\bar{n}|+\sum_{j=1}^{J} \delta_{r_{j}+\bar{n}_{j}+k_{j}, 0} \leq|n|+J-Z\left(n_{0}\right) \leq s-|r+\bar{n}+k|,
$$

which imply

$$
k_{j}+\delta_{r_{j}+\bar{n}_{j}+k_{j}, 0} \leq l-r_{j} \text { for all } 1 \leq j \leq J
$$

and

$$
|k|+Z(r+\bar{n}+k) \leq l J-|r|,
$$

respectively. Since (5.20) follows from (5.19), it does not constitute an independent bound. Furthermore, if $l \geq 1$, as is the case in (4.19) of Theorem 4.2, then (5.19) is equivalent to

$$
k_{j} \leq l-r_{j} \text { for all } 1 \leq j \leq J
$$

which becomes a new family of upper bounds for the $k_{j}$ summation in (5.6).

The new family of upper bounds for the $r_{j}$ summation in (5.6) which results from (5.21) and $k_{j} \geq 0$ is

$$
r_{j} \leq l \text { for all } 1 \leq j \leq J
$$

(which was already clear from (5.16)).

(5.7) now follows from (5.6), (5.16), (5.17), (5.18), (5.21), and (5.22).

6. Specialization to the conventional case. Unlike the intrinsic symbol, the conventional symbol of a pseudodifferential operator $A \in L^{l}(M, E, E)$ is dependent on a choice of local coordinate chart for $M$ and local frame for $E$. Such a choice on an open set $U \subset M$ provides one with a bijection $\psi: C^{\infty}\left(V, \mathbf{K}^{c}\right) \rightarrow \Gamma^{\infty}(E \mid U)$, where $V \subset \mathbf{R}^{d}$ is the image of $U$ under the coordinate diffeomorphism, $\mathbf{K}=\mathbf{R}$ or $\mathbf{C}$ according as $E$ is real or complex, and $c$ is the fiber dimension. The conventional symbol of $A$ with respect to this choice is defined as the (conventional) symbol of the induced operator $A^{\psi}=\psi^{-1} \circ A \circ \psi: C_{0}^{\infty}\left(V, \mathbf{K}^{c}\right) \rightarrow C^{\infty}\left(V, \mathbf{K}^{c}\right)$, which is simply the expression of $A$ in terms of the base and fiber coordinates. All of this makes no reference to (and is possible without) given connections on $T^{*}(M)$ and $E$. However, there exist natural flat connections on $T^{*}(V)=V \times \mathbf{R}^{d}$ and the trivial bundle $V \times \mathbf{K}^{c}$ over $V$, and if these are used in (3.2) then the intrinsic symbol of $A^{\psi}$ is equal to its conventional symbol (modulo smoothing). Thus the results of $\S 4$ contain as special cases results concerning the conventional symbolic calculus. In particular, we have the following version of Theorem 4.2:

THEOREM 6.1. Let $A \in L^{l}(M, E, E)$ be a differential operator of order $l>0$ which is elliptic with respect to a ray $\Gamma$ in $\mathbf{C}$, let $\lambda \in \Gamma$, and let $B_{\lambda} \in L^{-l}(M, E, E)$ be a resolvent parametrix of $A$. If the expression of $A$ relative to a choice of local coordinate chart for an open set $U \subset M$ and frame for $E \mid U$ is $A^{\psi}=\sum_{r=0}^{l} A_{r}^{\psi} \partial^{l-r}$, 
where $A_{r}^{\psi} \in C^{\infty}\left(V, G L(c, \mathbf{K}) \otimes S\left(\bigotimes^{l-r} \mathbf{R}^{d}\right)\right)(V$ is the image of $U$ under the coordinate diffeomorphism, $\mathbf{K}=\mathbf{R}$ or $\mathbf{C}$ according as $E$ is real or complex, and $c$ is the fiber dimension), then the conventional symbol of $B_{\lambda}$ with respect to this same choice of chart and frame has asymptotic expansion $b \sim \sum_{s \geq 0} b_{s}$, where $b_{0}=\left(A_{0}^{\psi}\left(\bigotimes^{l}(i \xi)\right)-\lambda\right)^{-1}$, and, for all $s \geq 1$,

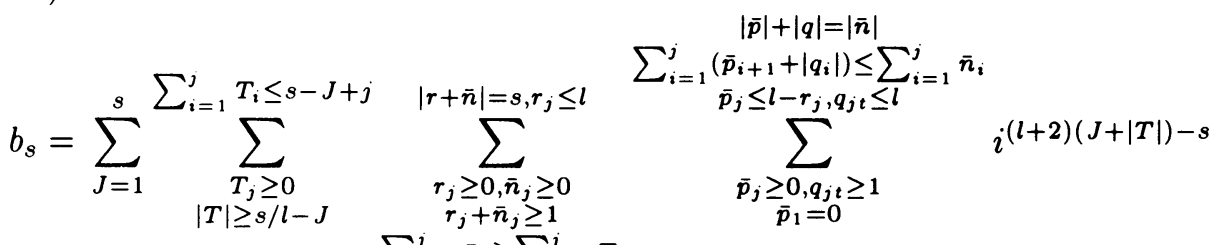

$$
\begin{aligned}
& \sum_{i=1}^{j} \bar{n}_{i} \geq \sum_{i=1}^{j} T_{i} \\
& \times(l !)^{|T|} \bar{N} !(l-r) !(\bar{n} ! \bar{p} ! q !(\bar{N}-\bar{n}) !(l-q) !(l-r-\bar{p}) !)^{-1} \\
& \times\left[\bigotimes_{j=1}^{J} R\left[\left(\bigotimes_{t=1}^{T_{j}} b_{0} A_{0}^{\psi}\right) b_{0}\left(\partial^{\bar{n}_{j}} A_{r_{j}}^{\psi}\right)\right]\right] b_{0}\left(\bigotimes^{l(J+|T|)-s} \xi\right) .
\end{aligned}
$$

In (6.1), all subscripted quantities are as listed in Definitions 4.3 and $4.4, l-r$ and $l-q$ are defined in Theorem 4.2, and the index contractions are described inductively as follows: For each $1 \leq j \leq J-1$, the $\bar{n}_{j}$ derivatives of the form $\partial^{\bar{n}_{j}}$ are combined with the $\sum_{i=1}^{j-1}\left(\bar{n}_{i}-\bar{p}_{i+1}-\left|q_{i}\right|\right)$ as yet uncontracted derivatives of the form $\partial^{\bar{n}_{i}}$ for all $1 \leq i \leq j-1$, and the total of

$$
\bar{N}_{j} \equiv \bar{n}_{j}+\sum_{i=1}^{j-1}\left(\bar{n}_{i}-\bar{p}_{i+1}-\left|q_{i}\right|\right)
$$

derivatives is symmetrized. Of these, $q_{j t}$ are contracted with $q_{j t}$ of the $l$ indices of the th $A_{0}^{\psi}$ factor in $\bigotimes_{t=1}^{T_{j}} b_{0} A_{0}^{\psi}$ for each $1 \leq t \leq T_{j}$, and $\bar{p}_{j+1}$ are contracted with $\bar{p}_{j+1}$ of the $l-r_{j+1}$ contravariant indices of $\partial^{\bar{n}_{j+1}} A_{r_{j+1}}^{\psi}$. The remaining

$$
\bar{N}_{j}-\bar{p}_{j+1}-\left|q_{j}\right|=\sum_{i=1}^{j}\left(\bar{n}_{i}-\bar{p}_{i+1}-\left|q_{i}\right|\right)
$$

derivatives are combined with the $\bar{n}_{j+1}$ derivatives of the form $\partial^{\bar{n}_{j+1}}$, and the process is repeated. After the final such operation (when $j=J-1$ ), the remaining

$$
\bar{N}_{J-1}-\bar{p}_{J}-\left|q_{J-1}\right|=\sum_{i=1}^{J-1}\left(\bar{n}_{i}-\bar{p}_{i+1}-\left|q_{i}\right|\right)
$$

derivatives are combined with the $\bar{n}_{J}$ derivatives of the form $\partial^{\bar{n}_{J}}$, and the total of

$$
\bar{N}_{J} \equiv \bar{n}_{J}+\sum_{i=1}^{J-1}\left(\bar{n}_{i}-\bar{p}_{i+1}-\left|q_{i}\right|\right)=\left|q_{J}\right|
$$

derivatives is symmetrized. Of these, $q_{J t}$ are contracted with $q_{J t}$ of the $l$ indices of the th $A_{0}^{\psi}$ factor in $\bigotimes_{t=1}^{T_{J}} b_{0} A_{0}^{\psi}$ for each $1 \leq t \leq T_{J}$. Finally, $\otimes^{l(J+|T|)-s} \xi$ is contracted with the remaining $l(J+|T|)-s$ uncontracted indices. 
PROOF. Since the connections on $T^{*}(V)=V \times \mathbf{R}^{d}$ and $V \times \mathbf{K}^{c}$ are chosen to be flat, $\nabla^{m} \Phi=0$ if $m \geq 2$ and $\nabla^{m} \tau^{V \times \mathbf{K}^{c}}=0$ if $m \geq 1$. Therefore the only nonvanishing terms in (4.19) are those for which $k_{j}=m_{j 0}=n_{j 0}=0$ for all $1 \leq j \leq J$. Hence $\left|m_{j}\right|=\left|n_{j}\right|=M_{j}=0, N_{j}=\bar{n}_{j}$, and $p_{j}=0$ for all $1 \leq j \leq J$, and (6.1) and its accompanying contractions follow.

REMARK 6.1. This result could also have been obtained directly from (4.8) and the product formula

$$
P_{u}(b, a)=i^{-u}(u !)^{-1}\left(D^{u} b\right)\left(\partial^{u} a\right),
$$

which is appropriate to the conventional calculus (cf. (3.5)).

REMARK 6.2. If desired, the summations over the variables $r_{j}$ and $\bar{n}_{j}$ in (6.1) may be ordered with the aid of (5.7):

$$
\sum_{\substack{r_{j} \geq 0, \bar{n}_{j} \geq 0 \\ r_{j}+\bar{n}_{j} \geq 1 \\ \sum_{i=1}^{j} \bar{n}_{i} \geq \sum_{i=1}^{j} T_{i}}}^{|r+\bar{n}|=s, r_{j} \leq l} T_{i} \sum_{r_{j} \geq 0}^{\substack{|r|+Z_{j}(T, r) \leq s \\ r_{j} \leq l}} \sum_{\bar{n}_{j} \geq 0}^{|\bar{n}|+Z_{j}(T-\bar{n}, r+\bar{n}) \leq s-|r|}[\cdots]
$$

Also, the summations over $J$ and $T_{j}$ may be reordered as described in Remark 4.7.

7. Concluding remarks. The theorems obtained in this paper reduce the determination of $b_{s}$ to the conceptually trivial problem of listing the terms specified by the multiple summations. Since the number of terms increases rapidly with $s$, hand calculations become impractical beyond the lowest orders. However, our results are well suited to machine calculation, and Fulling is developing computer programs to handle $b_{s}$ at relatively large $s$. (Descriptions and applications of these programs will be published elsewhere.) One anticipates that the greatest utility of the computer will arise not in printing out a multipage formula for $b_{s}$, but rather in generating $b_{s}$ internally as input into the computation of index formulas, effective actions, etc., for which the final results will be more compact.

It should be noted that many terms vanish in special cases of interest. For example, any term containing $\nabla \nabla \Phi$ disappears if the connection is torsionless. Also, any term containing a covariant derivative of $A_{0}$ vanishes if $A_{0}$ is constructed from a metric tensor with which the connection is compatible, as is ordinarily the case for the operators which arise in gravitational physics. Of course, many terms combine if $A_{0}$ (and hence $b_{0}$ ) is scalar.

It is desirable in many applications to express the covariant derivatives of $\Phi$ and $\tau^{E}$ in terms of the manifold curvature and torsion and the bundle curvature. As noted earlier (see Remark 2.5), this may be done recursively in several ways. Again, in high-order calculations it is most sensible to automate the process, and work in this direction is under way [Christensen (work in progress), Fulling 51].

In this paper we have focused our attention on the symbol of a resolvent parametrix. The corresponding integral kernel is obtainable, in principle, from the oscillatory integral (3.3). However, the symbol itself is sufficient to obtain the asymptotic expansion of the corresponding heat kernel (on the diagonal), and this will be our task in later papers. 


\section{REFERENCES}

1. M. F. Atiyah, R. Bott, and V. K. Patodi, On the heat equation and the index theorem, Invent. Math. 19 (1973), 279-330.

2. R. Balian and C. Bloch, Distribution of eigenfrequencies for the wave equation in a finite domain. I, Ann. Physics 60 (1970), 401-447; erratum ibid. 84 (1974), 559.

3. __ Distribution of eigenfrequencies for the wave equation in a finite domain. II, Ann. Physics 64 (1971), 271-307; erratum ibid. 84 (1974), 559-562.

4. H. P. Baltes and E. R. Hilf, Spectra of finite systems, Bibliographisches Institut, Mannheim, 1976.

5. N. H. Barth and S. M. Christensen, Quantizing fourth order gravity theories: the functional integral, Phys. Rev. D 28 (1983), 1876-1893.

6. J. Bokobza-Haggiag, Opérateurs pseudo-différentiels sur une variété différentiable, Ann. Inst. Fourier (Grenoble) 19 (1969), 125-177.

7. T. S. Bunch and L. Parker, Feynman propagator in curved spacetime: a momentum-space representation, Phys. Rev. D 20 (1979), 2499-2510.

8. C. Callias and C. H. Taubes, Functional determinants in Euclidean Yang-Mills theory, Comm. Math. Phys. 77 (1980), 229-250.

9. S. M. Christensen, Vacuum expectation value of the stress tensor in an arbitrary curved background: the covariant point-separation method, Phys. Rev. D 14 (1976), 2490-2501.

10. __ Regularization, renormalization, and covariant geodesic point-separation, Phys. Rev. D 17 (1978), 946-963.

11. _ Q Quantizing fourth order gravity theories, Quantum Structure of Space and Time (M. J. Duff and C. J. Isham, eds.), Cambridge Univ. Press, Cambridge, 1982, pp. 71-86.

12. C. Clark, The asymptotic distribution of eigenvalues and eigenfunctions for elliptic boundary value problems, SIAM Rev. 9 (1967), 627-646.

13. E. Cremmer and J. Scherk, Dual models in four dimensions with internal symmetries, Nuclear Phys. B 103 (1976), 399-425.

14. B. S. DeWitt, Dynamical theory of groups and fields, Gordon and Breach, New York, 1965.

15. H. Donnelly, Symmetric Einstein spaces and spectral geometry, Indiana Univ. Math. J. 24 (1974), 603-606.

16. L. Drager, On the intrinsic symbol calculus for pseudo-differential operators on manifolds, Ph.D. Dissertation, Brandeis University, 1978.

17. E. Getzler, Pseudodifferential operators on supermanifolds and the Atiyah-Singer index theorem, Comm. Math. Phys. 92 (1983), 163-178.

18. P. B. Gilkey, The spectral geometry of a Riemannian manifold, J. Differential Geom. 10 (1975), $601-618$.

19. __ The index theorem and the heat equation, Publish or Perish, Boston, 1974.

20. _ Invariance theory, the heat equation, and the Atiyah-Singer index theorem, Publish or Perish, Wilmington, 1984.

21. P. B. Gilkey and L. Smith, The eta invariant for a class of elliptic boundary value problems, Comm. Pure Appl. Math. 36 (1983), 85132.

22. P. Greiner, An asymptotic expansion for the heat equation, Arch. Rational Mech. Anal. 41 (1971), 163218.

23. J. Hadamard, Lectures on Cauchy's problem in linear differential equations, Dover, New York, 1952.

24. S. Helgason, Differential geometry, Lie, groups, and symmetric spaces, Academic Press, New York, 1978.

25. L. Hörmander, The spectral function of an elliptic operator, Acta Math. 121 (1968), 193-218.

26. M. Kac, Can one hear the shape of a drum?, Amer. Math. Monthly 73 (1966), 1-23.

27. G. Kennedy, The intrinsic symbolic calculus of pseudodifferential operators on manifolds (in preparation).

28. S. Kobayashi and K. Nomizu, Foundations of differential geometry, Vol. 1, Wiley, New York, 1963.

29. H. Kumano-Go, Pseudo-differential operators, M.I.T. Press, Cambridge, Mass., 1982.

30. J. F. Luciani, Space-time geometry and symmetry breaking, Nuclear Phys. B 135 (1978), 111 130. 
31. S. Minakshisundaram, Eigenfunctions on Riemannian manifolds, J. Indian Math. Soc. 17 (1953), 159-165.

32. S. Minakshisundaram and $\AA$. Pleijel, Some properties of the eigenfunctions of the Laplaceoperator on Riemannian manifolds, Canad. J. Math. 1 (1949), 242-256.

33. T. A. Osborn and R. Wong, Schrödinger spectral kernels: high order asymptotic expansions, J. Math. Phys. 24 (1983), 1487-1501.

34. L. Parker, Aspects of quantum field theory in curved spacetime: effective action and energymomentum tensor, Recent Developments in Gravitation: Cargèse 1978 (M. Levy and S. Deser, eds.), Plenum, New York, 1979, pp. 219-273.

35. B. E. Petersen, Introduction to the Fourier transform and pseudo-differential operators, Pitman, Boston, 1983.

36. T. Sakai, On eigen-values of Laplacian and curvature of Riemannian manifold, Tôhoku Math. J. 23 (1971), 589-603.

37. R. Schrader and M. E. Taylor, Small $\hbar$ asymptotics for quantum partition functions associated to Yang-Mills potentials, Comm. Math. Phys. 92 (1984), 555-594.

38. J. Schwinger, On gauge invariance and vacuum polarization, Phys. Rev. 82 (1951), 664-679.

39. R. T. Seeley, Complex powers of an elliptic operator, Singular Integrals, Proc. Sympos. Pure Math., Vol. 10, Amer. Math. Soc., Providence, R. I., 1967, pp. 288-307.

40. L. Smith, The asymptotics of the heat equation for a boundary value problem, Invent. Math. 63 (1981), 467-493.

41. M. E. Taylor, Pseudodifferential operators, Princeton Univ. Press, Princeton, 1981.

42. F. Treves, Introduction to pseudodifferential and Fourier integral operators, Vol. 1, Plenum, New York, 1980.

43. R. M. Wald, The back reaction effect in particle creation in curved spacetime, Comm. Math. Phys. 54 (1977), 1-19.

44. __ On the trace anomaly of a conformally invariant quantum field in curved spacetime, Phys. Rev. D 17 (1978), 1477-1484.

45. __ On the Euclidean approach to quantum field theory in curved spacetime, Comm. Math. Phys. 70 (1979), 221-242.

46. H. Widom, Families of pseudodifferential operators, Topics in Functional Analysis (I. Gohberg and M. Kac, eds.), Academic Press, New York, 1978, pp. 345-395.

47. $\ldots$ A complete symbolic calculus for pseudodifferential operators, Bull. Sci. Math. 104 (1980), 19-63.

48. __ Asymptotic expansions for pseudodifferential operators on bounded domains, Lecture Notes in Math., Vol. 1152, Springer-Verlag, Berlin, 1985.

49. S. F. J. Wilk, Y. Fujiwara, and T. A. Osborn, $N$-body Green's functions and their semi-classical expansion, Phys. Rev. A 24 (1981), 2187-2202.

50. E. Witten, Search for a realistic Kaluza-Klein theory, Nuclear Phys. B 186 (1981), 412-428.

\section{REFERENCES ADDED IN PROOF}

51. S. A. Fulling, The analytic approach to recursion relations, J. Symbolic Comput. (in press).

52. A. Ya. Rodionov and A. Yu. Taranov, Computation of covariant derivatives of the geodetic interval with coincident arguments, Classical Quantum Gravity 4 (1987), 1767-1775.

53. R. Schimming, Lineare Differentialoperatoren zweiter Ordnung mit metrischem Hauptteil und die Methode der Koinzidenzwerte in der Riemannschen Geometrie, Beitr. Anal. 15 (1981), 77-91.

\section{Department of Mathematics, Texas A\&M University, College Station, TEXAS 77843}

Current address (G. Kennedy): Department of Mathematics, University of California, Santa Cruz, California 95064 